\title{
A Quadrature-Free Method for Simulation and Inversion of 1.5D Direct Current (DC) Borehole Measurements
}

\author{
Sergio Rojas • Ignacio Muga • David Pardo
}

Received: date / Accepted: date

\begin{abstract}
Resistivity inverse problems are routinely solved in order to characterize hydrocarbon bearing formations. They often require a large number of forward problems simulations. When considering a one dimensional (1D) planarly layered media, semi-analytical methods can be employed in order to solve a single forward problem in a fraction of a second. However, in some situations, a large number of (over one million) simulations is required, preventing this method to be used as a real time (logging) alternative. In this paper, we propose a novel semi-analytical method that dramatically reduces the total computational time, so it can be employed for real time inversion. In our proposed method, we select an ad-hoc basis representation for the spectral solution such that its inverse Hankel transform can be computed analytically. The proposed method requires a pre-process that is expensive when compared with a single evaluation in classical semianalytical methods. However, subsequent evaluations can be rapidly obtained, decreasing thus the total computational time by orders of magnitude when the number of required forward simulations is large.
\end{abstract}

\section{S. Rojas}

Facultad de Ingeniería, Pontificia Universidad Católica de Chile, and Centro de Análisis Estocástico y Aplicaciones (ANESTOC), Pontificia Universidad Católica de Chile.

E-mail: srojash@gmail.com

I. Muga

Instituto de Matemáticas, Pontificia Universidad Catótica de Valparaíso, Chile.

E-mail: ignacio.muga@pucv.cl

D. Pardo

University of the Basque Country (UPV/ EHU), Basque Center for Applied Mathematics (BCAM), and IKERBASQUE, Bilbao, Spain.

E-mail: dzubiaur@gmail.com
Keywords Borehole measurements · Planarly layered media · Hankel transform - Quadrature-free method.

\section{Introduction}

The main objective in resistivity borehole measurements is to identify and characterize hydrocarbon (oil and gas) bearing formations. Maps of the Earth subsurface can be delineated by interpreting resistivity measurements obtained from various electromagnetic impulses in the media. From the mathematical point of view, one needs to solve an inverse problem in terms of the resisitivities, which requires simulation of a large number of forward problems. At the same time, in geosteering and logging-while-drillling operations, it is necessary to estimate the resistivities as fast as possible, ideally in real (logging) time (see [7,18]). Developing fast and robust numerical methods for simulation and inversion of resisitivity borehole measurements is still today a real challenge for the scientific community (see $[6,7,13,18])$.

Multiple researchers have developed three-dimensional (3D) numerical simulators of borehole resistivity measurements (e.g. [1,3,4,6,12-14,17,19,20,25-27]). These simulators have been successfully employed to study an quantify different physical effects occurring in 3D geometries. Since these solvers require solutions of 3D partial differential equations, they often entail an elevated computational cost, making them unfeasible for real time simulations.

In order to speed up computations in the inversion procedure, oil and oil-service companies often base some of their computations on a reduced model composed of a one dimensional (1D) planar isotropic or transversely isotropic (TI) layered media with unknown piece-wise 
constant resistivities. When considering a 3D source, the advantage of this model reduction is that $1.5 \mathrm{D}$ semi-analytical solutions (coming from a problem with $1 \mathrm{D}$ materials and 3D sources [21]) can be rapidly computed.

The traditional 1.5D semi-analytic technique consists of the following steps. First, we apply a Hankel transform, which is equivalent to applying two Fourier transforms along the invariant axes, leading to a 1D Helmholtz ordinary differential equation (ODE) for each Hankel frequency. Then, the solution of each 1D equation is computed analytically. Finally, the inverse Hankel transform is estimated by employing an ad-hoc quadrature (see [2, 8, 24], and more recently [9, 22, 28]). Using this method, a $1.5 \mathrm{D}$ forward simulation can be performed in a fraction of a second.

A considerable number of inversion methods based on the use of the aforementioned semi-analytical 1.5D method are available in the literature (eg. $[7,10,11,18])$. However, when dealing with an inverse problem, a large number (even millions) of $1.5 \mathrm{D}$ forward simulations may be required to account for the multiple logging positions, transmitters, frequencies, inverse iterations, and evaluations required for the computation of the Jacobian matrix (derivatives with respect to the material properties). More precisely, when $1.5 \mathrm{D}$ semi-analytical methods are used for the simulation of resitivity borehole measurements, the computational cost is proportional to the product of the number of layers, positions, transmitters, receivers and quadrature points. Moreover, Jacobian estimations (when using a numerical approximation for a single derivative) can adversely affect the computational cost, which sometimes prevents the method to be used as a real time alternative. This motives the study of designing faster semi-analytical methods for forward simulations and estimation of the Jacobian matrix.

In this work, we consider a direct current (DC) source embedded in a planar isotropic layered media. A DC formulation is employed to approximate measurements obtained by borehole logging instruments operating at very low frequencies, which can be numerically treated as a zero-frequency problem. The extension to TI media is straightforward (by introducing specific changes of coordinates in the horizontal directions), but we skip it here for the sake of simplicity. The DC case is also selected in this study for simplicity.

The main contribution of this work is the development of a novel semi-analytical method for the fast simulation and inversion of 1D DC resisivity borehole measurements. The proposed method, that we denote as Explicit Inverse Hankel Transform Method (EIHTM), consists of representing the ODE Hankel solution in terms of an exponential basis whose inverse Hankel transform can be computed analytically. Since quadrature approximations are not required, this is in fact a quadrature-free semi-analytical method, and this is the main difference with respect to classical methods. The proposed method consists of two steps:

- An initial pre-process that depends on the geometry (explicitly on the number of layers), and the number of basis terms.

- A post-process that only depends on the number of basis terms, positions, transmitters and receivers. In particular, this step is independent of the number of layers on the subsurface.

The computational cost of the proposed EIHTM also involves two terms:

- The first one corresponds to the pre-processing step, which is only performed once, since it is independent of the tool configuration and number of positions. Therefore, for a sufficiently large number of right hand sides (tool positions), this cost becomes negligible.

- The second term is related to an evaluation procedure. It is independent of the number of layers, and it is proportional to the product of exponential basis terms, positions, transmitters and receivers. Consequently, it is significantly cheaper than traditional quadrature-based semi-analytical method.

The proposed EIHTM is not intended to be competitive for a single solution evaluation. However, for a large number of right hand sides (logging positions), the total computational cost of the proposed EIHTM is significantly cheaper than the cost associated to classical methods. The method also allows estimations of the derivatives in an analogous way, and it can be easily implemented.

The remainder of this paper is organized as follows. First, we describe the principal aspects related to the resolution of the forward problem and Jacobian estimation by using a classical semi-analytical method. We also describe its computational cost estimates. Second, we formulate our proposed semi-analytical method. We also provide computational cost estimates and alternative formulas for the estimation of the derivatives with respect to the resistivity. Third, we perform several numerical experiments to illustrate the efficiency of the proposed method and validate the computational cost estimates. Finally, we conclude emphasizing the main advantages and limitations of the proposed method. 


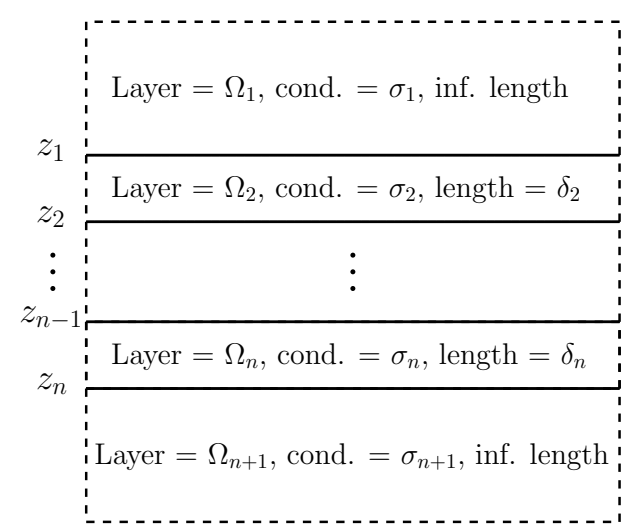

(a) $x z$ cross-section with media properties.

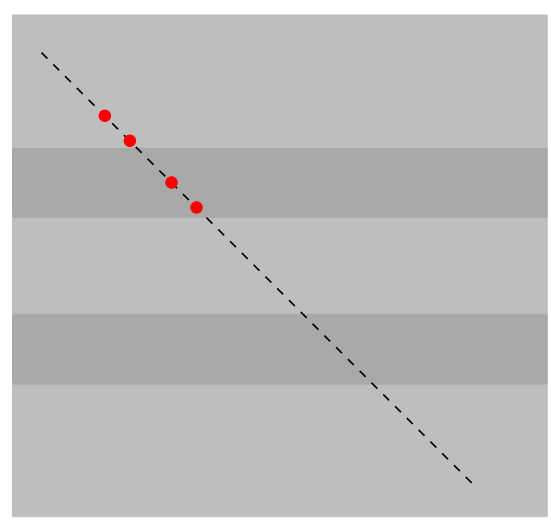

(b) Reduced model for a logging instrument with a two transmitters ( $\mathrm{Tx}$ ) and two receivers $(\mathrm{Rx})$ configuration, and a dip angle of $45^{\circ}$.

Fig. 1: Planar isotropic layered media.

\section{Model Problem}

We consider the full space $\mathbb{R}^{3}$ as an unbounded planar isotropic layered media characterized by $n+2$ vertical positions:

$-\infty=: z_{n+1}<z_{n}<\cdots<z_{1}<z_{0}:=+\infty$,

such that, for $i=1, \ldots, n+1$, the $i$-th layer is given by

$\Omega_{i}:=\left\{(x, y, z) \in \mathbb{R}^{3}: z_{i}<z<z_{i-1}\right\}$.

The conductivity tensor (respectively the resistivity tensor) of the $i$-th isotropic layer is characterized by a positive constant $\sigma_{i}$ (respectively $\rho_{i}:=1 / \sigma_{i}$ ), and it must be understood as the diagonal tensor obtained from multiplying this constant by the $3 \times 3$ identity matrix. For $i=2, \ldots, n$, the vertical length of the $i$-th layer is defined as $\delta_{i}=z_{i-1}-z_{i}$ (see Figure 1(a)).

A logging instrument is equipped with several transmitters $(\mathrm{Tx})$ and receivers $(\mathrm{Rx})$ located along a trajectory that crosses the 1D layered media (see Figure 1(b)). The effects of the mandrel and borehole are ignored, as it is customary in 1.5D simulations [24]. We also assume a DC source configuration, which is a good approximation for borehole logging instruments operating at very low frequencies.

DC resistivity logging applications are governed by the continuity equation at zero frequency, given by:

$-\operatorname{div}(\sigma \nabla u)=\operatorname{div} \mathbf{J}$,

where $\mathbf{J}$ denotes a given current source, $\sigma$ is a piece-wise constant discontinuous function which takes exactly the value $\sigma_{i}$ in $\Omega_{i}$, and $u$ is the electrostatic scalar potential, that is related to the electric field $\mathbf{E}$ by:

$\mathbf{E}=-\nabla u$.
Since the scalar potential and its flux should be globally continuous, the following conditions should be satisfied on the interfaces (see [23]):

$$
\begin{aligned}
{[u]_{i} } & =0, \text { for } i=1, \ldots, n \\
{\left[\sigma \partial_{z} u\right]_{i} } & =0, \text { for } i=1, \ldots, n,
\end{aligned}
$$

where $[\cdot]_{i}$ denotes the jump function defined at interface $\Gamma_{i}:=\left\{(x, y, z) \in \mathbb{R}^{3}: z=z_{i}\right\}$.

When a $3 \mathrm{D}$ point source is considered, the term $\operatorname{div} \mathbf{J}$ is mathematically modeled as a Dirac delta distribution. Due to the invariance with respect to $x$ and $y$ coordinates, we can assume without any loss of generality that the source is located at point $\left(0,0, z_{\mathrm{Tx}}\right)$. Then, Equation (2) becomes:

$$
-\operatorname{div}(\sigma \nabla u)=\delta(x) \delta(y) \delta\left(z-z_{\mathrm{Tx}}\right) .
$$

The postprocessed measurement recorded by the borehole instrument, denoted by $m(u)$, is given by a linear combination of the voltages recorded at the receivers. This linear combination depends on the tool configuration and it is designed in a manner that, when a homogeneous isotropic media is assumed, $m(u)$ coincides with the formation resistivity. For example, in the case of a tool configuration given by one transmitter (located at $\mathrm{Tx}$ ), and two receivers (located at $\mathrm{Rx}_{1}$ and $\mathrm{Rx}_{2}$ respectively), the measurement is defined as:

$m(u)=u\left(\mathrm{Rx}_{1}\right)-C_{f} u\left(\mathrm{Rx}_{2}\right)$,

where $C_{f}$ is known as the tool (correction) factor and it is given by:

$C_{f}=\frac{d\left(\mathrm{Tx}, \mathrm{Rx}_{2}\right)}{d\left(\mathrm{Tx}, \mathrm{Rx}_{1}\right)}-4 \pi d\left(\mathrm{Tx}, \mathrm{Rx}_{2}\right)$,

with $d(\cdot, \cdot)$ denoting the Euclidean distance function in $\mathbb{R}^{3}$. It can be easily verified that in a homogeneous 
media with isotropic resistivity $\rho_{h}:=1 / \sigma_{h}$, we have $m(u)=\rho$, since the electrostatic potential at Rx due to a point source located at $\mathrm{Tx}$, is given by [24]:

$u(\mathrm{Rx})=\frac{1}{4 \pi \sigma d(\mathrm{Tx}, \mathrm{Rx})}$.

Similar postprocessing formulas are derived when considering other logging instrument configurations.

\section{Traditional Hankel-based Solution Method}

\subsection{The Hankel Based Method}

In the second part of the last century, several researches developed 1.5D semi-analytical solutions for the electromagnetic field radiated by a magnetic dipole in a planarly layered media. See, for example, Wait [24] for isotropic formations, and Chew [2] and Kong [8] for TI formations. The traditional 1.5D semi-analytical technique (adapted to DC problems) is derived by following these steps:

- To perform a Hankel transform in the horizontal plane. This is equivalent to apply two Fourier transforms, reducing the original partial differential equation (5) into an ODE problem (in terms of the vertical variable $z$ ), where the dependency for each Hankel frequency is explicitly defined.

- The resulting ODE is solved analytically in terms of transmission and reflection coefficients that enforce the interface conditions (4) among consecutive layers.

- The final solution is computed by numerically evaluating the inverse Hankel transform of the above spectral solution.

\subsection{Computation of Derivatives}

To estimate the resisitivities in a layered media, it is necessary to solve an inverse problem. This basically consists of finding the global minimum of a non-linear least squares problem. Gradient based methods such as Gauss-Newton are often employed for such purpose (see [18]). Each iteration in the inversion procedure requires, for a sufficiently large number of tool positions, the resolution of a forward problem and a computation of the Jacobian matrix (derivatives with respect to the resistivities). Since a fast estimation and an easy implementation are desirable, the following estimation of the conductivity derivative (based on its definition) is often employed to compute the Jacobian matrix:

$\partial_{\sigma_{j}} m(u) \approx \frac{m\left(u_{\sigma_{j}+h}\right)-m(u)}{h}$, where $h>0$ is a pre-defined (sufficiently small) constant, and $u_{\sigma_{j}+h}$ corresponds to the solution obtained from problem (5) after substituting $\sigma_{j}$ by $\sigma_{j}+h$. The respective Jacobian matrix is obtained by applying the chain rule.

As an alternative to approximation (9), it is also possible to compute the Jacobian matrix using semianalytical formulas as those shown in [22]. This approach performs more accurate approximations and does not require a pre-defined parameter $h$. However, it requires a more complex implementation and its computational cost is unclear (and not reported in [22]).

\subsection{Computational Cost Estimates}

When using a traditional 1.5D semi-analytical method to solve a forward problem (e.g. $[2,9,22]$ ), the computational cost of a single evaluation of the solution $\left(T_{\mathrm{se}}\right)$ is estimated as:

$T_{\text {se }}=\mathcal{O}\left(N_{\text {layer }} \cdot N_{\text {quad }}\right)$,

$N_{\text {layer }}$ is the number of layers and $N_{\text {quad }}$ is the number of quadrature points used in the Hankel inversion procedure. A full DC forward simulation requires the solution evaluated at all logging positions. Thus the total cost $\left(T_{\mathrm{fe}}\right)$ involves the cost of a single evaluation, multiplied by the number of positions $\left(N_{\text {pos }}\right)$, transmitters $\left(N_{\mathrm{Tx}}\right)$, and receivers $\left(N_{\mathrm{Rx}}\right)$, i.e.

$T_{\mathrm{fe}}=N_{\mathrm{pos}} \cdot N_{\mathrm{Tx}} \cdot N_{\mathrm{Rx}} \cdot T_{\mathrm{se}}$

When approximation (9) is used to calculate the Jacobian matrix, it is necessary to solve $N_{\text {layer }}$ auxiliary problems (one per layer resistivity). Therefore, the cost to compute the Jacobian matrix $\left(T_{\mathrm{jac}}\right)$ is given by:

$T_{\text {jac }}=N_{\text {layer }} \cdot T_{\text {fe }}$

Finally, denoting by $N_{\text {iter }}$ the number of iterations used by the iterative algorithm, the cost of the inversion procedure $\left(T_{\mathrm{inv}}\right)$ is approximately:

$T_{\text {inv }}=\mathcal{O}\left(N_{\text {iter }} \cdot N_{\text {layer }} \cdot T_{\text {fe }}\right)$.

Even if a forward simulation can be performed in a fraction of a second, the total time of the inversion may be prohibitively expensive in order to perform real (logging) time inversion. This motivates the study of novel methods focused on reducing $T_{\mathrm{fe}}$.

In the following, we present a novel method with a lower $T_{\text {fe }}$ (when $N_{\text {pos }}$ is sufficiently large), so the inversion can be efficiently performed. 


\section{The Explicit Inverse Hankel Transform Method (EIHTM)}

As in classical 1.5D semi-analytical methods, the EIHTM recovers the potential $u$ satisfying (4)-(5) by applying a Hankel transform over the invariant axis. To do this, in the EIHTM, the potential $u$ is first split into a sum of a known primary field $u_{p}$ satisfying (5), and a secondary field $u_{s}$ to be determined. Then, a Hankel transform is applied to the equations that must be satisfied by $u=u_{p}+u_{s}$, namely equations (5) and (4). The Hankel transform of $u_{s}$ (denoted by $\hat{u}_{s}$ ) is obtained analytically as a layer-by-layer representation given by:

$$
\begin{aligned}
\left.\hat{u}_{s}\left(\xi, z, z_{\mathrm{Tx}}\right)\right|_{\Omega_{i}} & =\frac{1}{4 \pi \xi} c_{i 1}\left(\xi, z_{\mathrm{Tx}}\right) e^{-\xi\left(z-z_{i}\right)} \\
& +\frac{1}{4 \pi \xi} c_{i 2}\left(\xi, z_{\mathrm{Tx}}\right) e^{-\xi\left(z_{i-1}-z\right)}
\end{aligned}
$$

where $\left.(\cdot)\right|_{\Omega_{i}}$ denotes the restriction to the $i$-th layer, $z_{i}, z_{i-1}$ are the $z$-limits of the layer (see Equation (1)), and $\xi \geq 0$ is the spectral Hankel frequency. The spectral functions $c_{12}, c_{(n+1) 1}$ are assumed to be equal to zero, while the rest of the spectral functions are obtained by forcing the transmission conditions (4). They are globally represented in a vectorial form as:

$\mathbf{c}\left(\xi, z_{\mathrm{Tx}}\right)=A^{-1}(\xi) \mathbf{r}\left(\xi, z_{\mathrm{Tx}}\right)$,

where $A(\xi)$ is a spectral matrix that does not depend on transmitters and receivers, and $\mathbf{r}\left(\xi, z_{\mathrm{Tx}}\right)$ represents the dependency of the primary field.

In classical $1.5 \mathrm{D}$ semi-analytical methods, the product $A^{-1}(\xi) \mathbf{r}\left(\xi, z_{\mathrm{Tx}}\right)$ is efficiently computed analytically, and the Hankel inversion is obtained by quadrature, making possible to obtain a single evaluation in a fraction of a second. However, when measurements at a large number of tool positions are required, the total computational cost can increase considerably (see section 4.5). In contrast, the EIHTM builds by design an approximation to the inverse matrix function $A^{-1}(\xi)$ (denoted by $\tilde{A}^{-1}(\xi)$ ) such that, when approximating (15) by:

$\tilde{\mathbf{c}}\left(\xi, z_{\mathrm{Tx}}\right)=\tilde{A}^{-1}(\xi) \mathbf{r}\left(\xi, z_{\mathrm{Tx}}\right)$,

the spectral solution $\hat{u}_{s}$ is approximated as:

$$
\begin{aligned}
\left.\hat{u}_{s}\left(\xi, z, z_{\mathrm{Tx}}\right)\right|_{\Omega_{i}} & \approx \frac{1}{4 \pi \xi} \tilde{c}_{i 1}\left(\xi, z_{\mathrm{Tx}}\right) e^{-\xi\left(z-z_{i}\right)} \\
& +\frac{1}{4 \pi \xi} \tilde{c}_{i 2}\left(\xi, z_{\mathrm{Tx}}\right) e^{-\xi\left(z_{i-1}-z\right)} \\
& =: \tilde{\hat{u}}_{i 1}\left(\xi, z, z_{\mathrm{Tx}}\right)+\tilde{\hat{u}}_{i 2}\left(\xi, z, z_{\mathrm{Tx}}\right),
\end{aligned}
$$

and its inverse Hankel transform can be computed analytically. Thus, in cylindrical coordinates $(r, \theta, z)$, the potential $u$ is approximated as:

$$
\begin{aligned}
\left.u\left(r, z, z_{\mathrm{Tx}}\right)\right|_{\Omega_{i}} & \left.\approx u_{p}\left(r, z, z_{\mathrm{Tx}}\right)\right|_{\Omega_{i}}+\tilde{u}_{i 1}\left(r, z, z_{\mathrm{Tx}}\right) \\
& +\tilde{u}_{i 2}\left(r, z, z_{\mathrm{Tx}}\right),
\end{aligned}
$$

where $\tilde{u}_{i 1}$ (respectively $\tilde{u}_{i 2}$ ) denotes the analytic inverse Hankel transform of $\tilde{\hat{u}}_{i 1}$ (respectively $\tilde{\hat{u}}_{i 2}$ ). The principal advantage of the EIHTM is that it is quadraturefree, since integral approximations are no longer required. The steps of the EIHTM are described in Algorithm 1. The EIHTM requires a pre-process for the

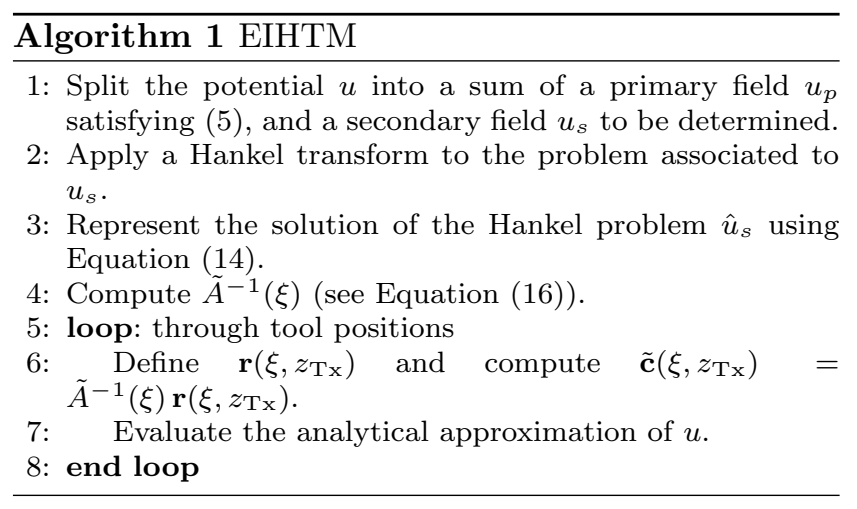

computation of $\tilde{A}^{-1}(\xi)$ (line 4 in Algorithm 1) that is expensive when comparing with a single evaluation in classical semi-analytical methods, but it has the advantage that it only needs to be estimated once. Then, for each tool position, its solution cost (lines 6, 7 in Algorithm 1) is independent of the number of layers. In the following, we describe in detail the steps of the EIHTM Algorithm.

\subsection{Decomposition into a primary and a secondary field}

We start by splitting the electrostatic potential $u$ into a sum of a primary field $u_{p}$ and a secondary field $u_{s}$. The primary field is a known potential satisfying (5), and the secondary field is an unknown potential satisfying the homogeneous equation:

$-\operatorname{div}\left(\sigma \nabla u_{s}\right)=0$,

and the interface conditions:

$$
\begin{aligned}
{\left[u_{s}\right]_{i} } & =-\left[u_{p}\right]_{i}, & \text { for } i=1, \ldots, n, \\
{\left[\sigma \partial_{z} u_{s}\right]_{i} } & =-\left[\sigma \partial_{z} u_{p}\right]_{i}, & \text { for } i=1, \ldots, n
\end{aligned}
$$

Due to the linear nature of the equations, it can be easily verified that $u=u_{p}+u_{s}$ corresponds to the desired potential satisfying (4)-(5).

\subsection{The discontinuous primary field}

A natural choice for the primary field satisfying (5) is the fundamental solution for a homogeneous isotropic 


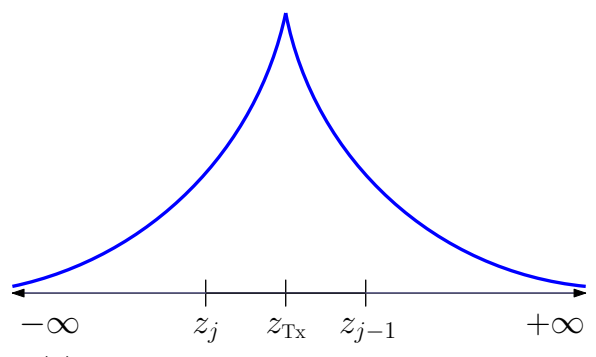

(a) Continuous fundamental solution.

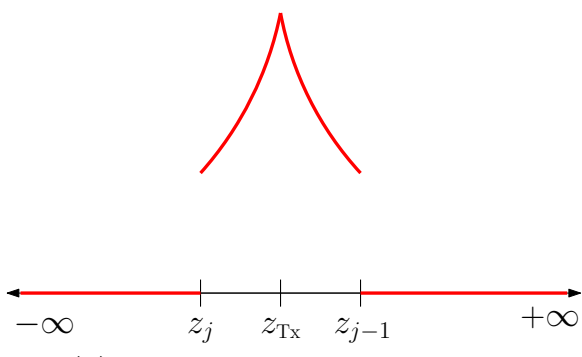

(b) Discontinuous primary field.

Fig. 2: Example of the primary field (in terms of $z$ ) selection for a fixed Hankel frequency $\xi>0$, and a source placed in a layer of finite length.

media, given by:

$u_{f}=\frac{1}{4 \pi \sigma_{\mathrm{Tx}} d(\mathrm{Tx}, \mathrm{Rx})}$,

where $\sigma_{\mathrm{Tx}}$ denotes the conductivity of the layer containing the source, and $d(\mathrm{Tx}, \mathrm{Rx})$ denotes the Euclidean distance between Tx and Rx. The spectral Hankel representation of (21) is given by:

$\hat{u}_{f}=\frac{e^{-\xi\left|z-z_{\mathrm{Tx}}\right|}}{4 \pi \sigma_{\mathrm{Tx}} \xi}$.

Since the field $\hat{u}_{f}$ is continuous (see Figure $2(\mathrm{a})$ ), the Dirichlet jumps in the right-hand side of equation (20) are always equal to zero, while the fluxes are not. The principal disadvantage of this choice is that the number of nonzero entries on the right-side term $\mathbf{r}\left(\xi, z_{\mathrm{Tx}}\right)$ (see Equation (15)) increases with the number of interfaces. Such dependency can be avoided by introducing the following discontinuous field:

$\left.u_{p}\right|_{\Omega_{j}}:=\left\{\begin{array}{cl}u_{f} & \text { if } j=i_{\mathrm{Tx}} \\ 0 & \text { if } j \neq i_{\mathrm{Tx}}\end{array}\right.$

where $i_{\mathrm{Tx}}$ corresponds to the index of the layer containing the source. Since the Hankel transform does not depend on $z$, the spectral representation of (23) is given by (see Figure 2(b)):

$\left.\hat{u}_{p}\right|_{\Omega_{j}}:=\left\{\begin{array}{cl}\hat{u}_{f} & \text { if } j=i_{\mathrm{Tx}} \\ 0 & \text { if } j \neq i_{\mathrm{Tx}} .\end{array}\right.$

With this selection, the right-hand side terms in equation (20) are different from zero only at the interfaces of the layer containing the source. This implies that the post-processing indicated on line 7 of Algorithm 1 (solution of the problem for a new tool position) is independent of the number of layers. Therefore, the primary field $u_{p}$ given by equation (23) is the one that we are going to use along this paper.

\subsection{The spectral Hankel problem}

As with the traditional $1.5 \mathrm{D}$ semi-analytic technique, we apply a Hankel transform to equations (19), (20), and solve the spectral ODE equation in terms of the vertical variable $z$ :

$\left(\xi^{2}-\partial_{z}^{2}\right) \hat{u}_{s}=0$

with the spectral interface conditions:

$\left[\hat{u}_{s}\right]_{i}=-\left[\hat{u}_{p}\right]_{i}, \quad$ for $i=1, \ldots, n$,

$\left[\sigma \partial_{z} \hat{u}_{s}\right]_{i}=-\left[\sigma \partial_{z} \hat{u}_{p}\right]_{i}$, for $i=1, \ldots, n$.

General solutions of equation (25) can be expressed as (14), where the $z$-independent coefficients $c_{i 1}\left(\xi, z_{\mathrm{Tx}}\right), c_{i 1}\left(\xi, z_{\mathrm{Tx}}\right)$ have to be determined by forcing the transmission conditions $(26)$ (recall that $c_{(n+1) 1}=$ $\left.c_{12}=0\right)$. We define:

$$
\begin{aligned}
\mathbf{c}= & \left(c_{11}, c_{21}, c_{22}, \ldots, c_{i 1}, c_{i 2}, c_{(i+1) 1}, c_{(i+1) 2},\right. \\
& \left.\ldots, c_{n 1}, c_{n 2}, c_{(n+1) 2}\right) .
\end{aligned}
$$

Coefficients of vector $\mathbf{c}$ are obtained by solving a linear system of the form:

$A(\xi) \mathbf{c}\left(\xi, z_{\mathrm{Tx}}\right)=\mathbf{r}\left(\xi, z_{\mathrm{Tx}}\right)$,

dictated by the interface conditions, with

$A(\xi)=\left[\begin{array}{cccccc}1 & -e_{2} & -1 & 0 & 0 & 0 \\ \sigma_{1} & -\sigma_{2} e_{2} & \sigma_{2} & 0 & 0 & 0 \\ \vdots & \vdots & \vdots & \vdots & \vdots & \vdots \\ 0 & 1 & e_{i} & -e_{i+1} & -1 & 0 \\ 0 & \sigma_{i} & -\sigma_{i} e_{i} & -\sigma_{i+1} e_{i+1} & \sigma_{i+1} & 0 \\ \vdots & \vdots & \vdots & \vdots & \vdots & \vdots \\ 0 & 0 & 0 & 1 & e_{n} & -1 \\ 0 & 0 & 0 & \sigma_{n} & -\sigma_{n} e_{n} \sigma_{n+1}\end{array}\right]$

where $e_{i}:=e^{-\xi \delta_{i}}$, with $\delta_{i}$ denoting the $i$-th vertical layer length (see Figure 1(a)) and, for $i=1, \ldots, n$, the 
right-hand side vector $\mathbf{r}$ is given by:

$$
\begin{aligned}
r_{2 i-1} & =-4 \pi \xi\left[\hat{u}_{p}\right]_{i}, \\
r_{2 i} & =-4 \pi \xi\left[\sigma \partial_{z} \hat{u}_{p}\right]_{i} .
\end{aligned}
$$

We consider the following cases for the right-hand side term (30):

- If $i_{\mathrm{Tx}}=1$, then $r_{k}=$

$$
\left\{\begin{array}{cl}
-\frac{1}{\sigma_{\mathrm{Tx}}} e^{-\xi\left|z_{1}-z_{\mathrm{Tx}}\right|} & \text { if } k=1 \\
\operatorname{sgn}\left(z_{1}-z_{\mathrm{Tx}}\right) e^{-\xi\left|z_{1}-z_{\mathrm{Tx}}\right|} & \text { if } k=2 \\
0 & \text { otherwise. }
\end{array}\right.
$$

- If $i_{\mathrm{Tx}}=n+1$, then $r_{k}=$

$$
\left\{\begin{array}{cl}
\frac{1}{\sigma_{\mathrm{Tx}}} e^{-\xi\left|z_{n}-z_{\mathrm{Tx}}\right|} & \text { if } k=2 n-1, \\
\operatorname{sgn}\left(z_{\mathrm{Tx}}-z_{n}\right) e^{-\xi\left|z_{n}-z_{\mathrm{Tx}}\right|} & \text { if } k=2 n \\
0 & \text { otherwise. }
\end{array}\right.
$$

- If $1<i_{\mathrm{Tx}}=j<n+1$, then $r_{k}=$

$$
\left\{\begin{array}{cl}
\frac{1}{\sigma_{\mathrm{Tx}}} e^{-\xi\left|z_{j-1}-z_{\mathrm{Tx}}\right|} & \text { if } k=2 j-3 \\
\operatorname{sgn}\left(z_{\mathrm{Tx}}-z_{j-1}\right) e^{-\xi\left|z_{j-1}-z_{\mathrm{Tx}}\right|} & \text { if } k=2 j-2 \\
-\frac{1}{\sigma_{\mathrm{Tx}}} e^{-\xi\left|z_{j}-z_{\mathrm{Tx}}\right|} & \text { if } k=2 j-1 \\
\operatorname{sgn}\left(z_{j}-z_{\mathrm{Tx}}\right) e^{-\xi\left|z_{j}-z_{\mathrm{Tx}}\right|} & \text { if } k=2 j \\
0 & \text { otherwise. }
\end{array}\right.
$$

Remark 1 The dependency of the source is explicitly given in the right-hand side term $\mathbf{r}\left(\xi, z_{\mathrm{Tx}}\right)$ (equations (31)-(33)), and the dependency of the receiver is explicitly given in the exponential terms of $\hat{u}_{s}$ (Equation (18)). Thus, matrix $A(\xi)$ (and its inverse) are independent of the tool position.

\subsection{The analytic Hankel inversion procedure}

We observe that the inverse Hankel transform of functions of the form:

$\hat{g}_{\lambda}(\xi)=t \frac{e^{-\xi \lambda}}{\xi}$, with $t \in \mathbb{R}, \lambda>0$,

can be computed analytically and is given by [15]:

$g_{\lambda}(r)=\frac{t}{\sqrt{r^{2}+\lambda^{2}}}$.

To obtain the spectral vector approximation $\tilde{\mathbf{c}}\left(\xi, z_{\mathrm{Tx}}\right)$ (see (16)), the EIHTM defines a priori $m+1$ nonnegative exponential coefficients, denoted by $\lambda_{k}$, such that $\lambda_{0}=0$ and $\lambda_{k}>0$, for $k=1, \ldots, m$. Then, it computes an approximation of matrix $A^{-1}(\xi)$, denoted by $\tilde{A}^{-1}(\xi)$, such that the $i j$-th matrix element of $\tilde{A}^{-1}(\xi)$, denoted by $\tilde{a}_{i j}^{-1}(\xi)$, is of the form:

$\tilde{a}_{i j}^{-1}(\xi)=\sum_{k=0}^{m} t_{k}^{i j} e^{-\xi \lambda_{k}}$,

where the unknown coefficients $t_{k}^{i j}, k=0, \ldots, m$ must be determined. By the exponential representation of the right-hand side vector $\mathbf{r}\left(\xi, z_{\mathrm{Tx}}\right)$ (see equations (31)(33)), it is clear that the inverse Hankel transform of approximation (17) can be computed analytically. For instance, if the source and the receiver are located inside layer $\Omega_{1}$, then $\tilde{\hat{u}}_{12}=0$ (since $\left.\tilde{c}_{12}=0\right)$, and

$$
\begin{aligned}
\tilde{\hat{u}}_{11} & =\frac{1}{4 \pi} \tilde{c}_{11}\left(\xi, z, z_{\mathrm{Tx}}\right) e^{-\xi\left(z-z_{1}\right)} \\
& =-\frac{1}{4 \pi} \sum_{k=0}^{m}\left(t_{k}^{12}+t_{k}^{11} / \sigma_{\mathrm{Tx}}\right) e^{-\xi\left(z+z_{\mathrm{Tx}}+\lambda_{k}-2 z_{1}\right)} .
\end{aligned}
$$

Thus, from equations (34), (35), we have:

$\left.u_{s}\right|_{\Omega_{1}} \approx-\frac{1}{4 \pi} \sum_{k=0}^{m} \frac{t_{k}^{12}+t_{k}^{11} / \sigma_{\mathrm{Tx}}}{\sqrt{r^{2}+\left(z+z_{\mathrm{Tx}}+\lambda_{k}-2 z_{1}\right)^{2}}}$.

In order to find $\tilde{A}^{-1}(\xi)$ such that $\mathbf{c}\left(\xi, z_{\mathrm{Tx}}\right) \approx$ $\tilde{A}^{-1}(\xi) \mathbf{r}\left(\xi, z_{\mathrm{Tx}}\right)$, we perform a left-side inverse matrix function approximation for $A^{-1}(\xi)$, i.e. we perform a spectral matrix approximation $\tilde{A}^{-1}(\xi)$ such that

$\tilde{A}^{-1}(\xi) A(\xi) \approx I_{d}$,

where $I_{d}$ denotes the $2 n \times 2 n$ identity matrix. In fact, multiplying the linear equation (28) by the left-side inverse matrix $\tilde{A}^{-1}(\xi)$, it holds:

$$
\begin{aligned}
\mathbf{c}\left(\xi, z_{\mathrm{Tx}}\right) & \approx \tilde{A}^{-1}(\xi) A(\xi) \mathbf{c}\left(\xi, z_{\mathrm{Tx}}\right) \\
& =\tilde{A}^{-1}(\xi) \mathbf{r}\left(\xi, z_{\mathrm{Tx}}\right) .
\end{aligned}
$$

To solve problem (39), we equivalently solve:

$A^{T}(\xi) \tilde{A}^{-T}(\xi) \approx I_{d}$,

where $(\cdot)^{T}$ denotes the transpose operator. To obtain an approximation of $\tilde{A}^{-T}(\xi)$, the following steps are performed:

- The constant dependency $\left(t_{0}^{i j}\right.$ coefficients $)$ is obtained analytically.

- The exponential coefficients $\lambda_{k}, k=1, \ldots, m$, are defined in terms of the layer lengths.

- The remaining $t_{k}^{i j}$ coefficients $(k=1, \ldots, m)$ are obtained by solving a least squares (LS) problem.

In the following, we explain in detail the previous procedure. 


\subsubsection{Constant dependency for the Inverse Matrix}

The constant dependency of the spectral matrix function $A^{-T}(\xi)$ can be obtained analytically by noting that $A^{T}(\xi)$ admits a decomposition (see Equation (29)):

$A^{T}(\xi)=C+D(\xi)$

with $C$ being a $\xi$-independent invertible matrix, and $D(\xi)=A^{T}(\xi)-C$ being a matrix with its entries expressed in terms of exponential functions.

Remark 2 We define $C=\lim _{\xi \rightarrow+\infty} A^{T}(\xi)$. From Equation (29), it is straightforward to see that $C$ is an invertible matrix, since the conductivitites $\sigma_{i}$ are positive.

If we write $A^{-T}(\xi)$ as:

$A^{-T}(\xi)=C^{-1}+Z(\xi)$,

then the unknown matrix $Z(\xi)$ must satisfy:

$A^{T}(\xi) Z(\xi)=-D(\xi) C^{-1}$.

By introducing the change of variables $Z(\xi)=$ $-Y(\xi) C^{-1}$, problem (44) is equivalent to find $Y(\xi)$ such that:

$A^{T}(\xi) Y(\xi)=D(\xi)$,

Summarizing, $A^{-T}(\xi)$ can be written as:

$A^{-T}(\xi)=C^{-1}-Y(\xi) C^{-1}$,

with $Y(\xi)$ being the unknown matrix function satisfying Equation (45).

Since the right-hand side matrix term in equation (45) has an exponentially decreasing behavior (when $\xi \rightarrow+\infty$ ), and $A^{T}(\xi)$ is invertible for all $\xi$ (with limit equal to $C$, when $\xi \rightarrow+\infty$ ), we can assume that $Y(\xi)=A^{-T}(\xi) D(\xi)$ can be accurately approximated by a matrix function $\tilde{Y}(\xi)$ with coefficients given by linear combinations of exponential functions of the form $e^{-\xi \lambda}$ (with $\lambda>0$ ). Then, the exponential approximation of $A^{-T}(\xi)$ is given by:

$\tilde{A}^{-T}(\xi)=C^{-1}-\tilde{Y}(\xi) C^{-1}$.

Note that with this reduction, the constant dependency of $\tilde{A}^{-T}(\xi)$ is given by $C^{-1}$, thus it does not need to be estimated.

\subsubsection{Exponential coefficients determinantion}

Matrix $D(\xi)$ in representation (42) admits the decomposition:

$D(\xi)=\sum_{k=2}^{n} e^{-\delta_{k} \xi} D_{k}$,

where, for $k=2, \ldots, n, D_{k}$ is a constant matrix, and $\delta_{k}$ the length of the $k$-th layer (see (29)). Denoting by $\delta_{\min }$ (respectively $\delta_{\max }$ ) the minimum (respectively maximum) finite layer length, we define (a priori) the following set of exponential coefficients with cardinality $m=N_{\min }+N_{\max }$ :

$$
\begin{aligned}
\Lambda= & \left\{\delta_{\min }, 2 \delta_{\min }, \ldots, N_{\min } \delta_{\min },\right. \\
& \left.\delta_{\max }, 2 \delta_{\max }, \ldots, N_{\max } \delta_{\max }\right\},
\end{aligned}
$$

and we assume that each element of matrix $\tilde{Y}(\xi)$ is a linear combination of the spectral functions $e^{-\lambda_{i} \xi}$, with $\lambda_{i} \in \Lambda$. The multiples of $\delta_{\min }$ are selected to reproduce the far field behavior $(\xi \rightarrow+\infty)$ of $D(\xi)$, while multiples of $\delta_{\max }$ are employed to act as correctors in the near field ( $\xi$ small). In principle, the number of required coefficients is unclear. However, we show by numerical examples that just a few number of them are needed to obtain accurate approximations.

\subsubsection{The Least Squares problem for the residual}

In vectorial form, problem (45) is equivalent to:

$A^{T}(\xi) \mathbf{y}_{i}(\xi)=\mathbf{d}_{i}(\xi), i=1, \ldots, 2 n$

where $\mathbf{y}_{i}(\xi), \mathbf{d}_{i}(\xi)$ denotes the $i$-th column of $Y(\xi)$ and $D(\xi)$, respectively.

Considering the vector of exponential coefficients $\boldsymbol{\lambda}$ defined in (49), we define the $N=2 \mathrm{~nm}$ basis elements:

$$
\begin{gathered}
\tilde{\mathbf{v}}_{1}:=e^{-\lambda_{1} \xi} \hat{\mathbf{e}}_{1}, \ldots, \tilde{\mathbf{v}}_{m}:=e^{-\lambda_{m} \xi} \hat{\mathbf{e}}_{1}, \\
\tilde{\mathbf{v}}_{m+1}:=e^{-\lambda_{1} \xi} \hat{\mathbf{e}}_{2}, \ldots, \tilde{\mathbf{v}}_{2 m}:=e^{-\lambda_{m} \xi} \hat{\mathbf{e}}_{2}, \\
\vdots \\
\tilde{\mathbf{v}}_{(2 n-1) m+1}:=e^{-\lambda_{1} \xi} \hat{\mathbf{e}}_{2 n}, \ldots, \tilde{\mathbf{v}}_{2 n m}:=e^{-\lambda_{m} \xi} \hat{\mathbf{e}}_{2 n},
\end{gathered}
$$

where $\hat{\mathbf{e}}_{j}$ denotes the canonical $j$-th basis vector of $\mathbb{R}^{2 n}$. For $i=1, \ldots, 2 n$, to obtain the exponential approximation of vector $\mathbf{y}_{i}(\xi)$ (denoted by $\tilde{\mathbf{y}}_{i}(\xi)$ ), we solve the following Least Squares problem :

Find $s_{i 1}, \ldots, s_{i N} \in \mathbb{R}$, such that

$\tilde{\mathbf{y}}_{i}(\xi)=\sum_{l=1}^{N} s_{i l} \tilde{\mathbf{v}}_{l}(\xi)$ minimizes $\left\|\mathbf{d}_{i}-A^{T} \tilde{\mathbf{y}}_{i}\right\|^{2}$,

where $\|\tilde{\mathbf{v}}\|^{2}=(\tilde{\mathbf{v}}, \tilde{\mathbf{v}})$, with $(\tilde{\mathbf{v}}, \tilde{\mathbf{w}})$ denoting the $\left[L^{2}\left(\mathbb{R}_{0}^{+}\right)\right]^{2 n}$ inner product given by

$(\tilde{\mathbf{v}}, \tilde{\mathbf{w}})=\int_{0}^{+\infty} \tilde{\mathbf{w}}^{T}(t) \tilde{\mathbf{v}}(t) d t$.

Defining $\mathbf{s}_{i}:=\left\{s_{i 1}, \ldots, s_{i N}\right\}^{T}$ in matricial form, minimization problem (52) is given by:

$M \mathbf{s}_{i}=\mathbf{h}_{i}$,

with

$M_{l, j}=\left(A^{T} \tilde{\mathbf{v}}_{j}, A^{T} \tilde{\mathbf{v}}_{l}\right)$, 
and

$\mathbf{h}_{i}=\left\{\left(\mathbf{d}_{i}, A^{T} \tilde{\mathbf{v}}_{1}\right), \ldots,\left(\mathbf{d}_{i}, A^{T} \tilde{\mathbf{v}}_{N}\right)\right\}^{T}$.

The Least Squares problem has the following properties:

- It provides the representation of $\tilde{\mathbf{y}}_{i}(\xi)$.

- The inner products $\left(A^{T} \tilde{\mathbf{v}}_{j}, A^{T} \tilde{\mathbf{v}}_{l}\right)$, and $\left(\mathbf{e}_{i}, A^{T} \tilde{\mathbf{v}}_{l}\right)$ are computed analytically.

- Matrix $M$ is sparse, symmetric, and is independent of the index $i=1, \ldots, 2 n$. Thus, it is constructed just once.

Remark 3 Note that the representation given for $\tilde{A}^{-1}(\xi)$ in (36) is obtained by using the factorization $h_{1} e^{-\xi \lambda_{i}}+h_{2} e^{-\xi \lambda_{i}}=\left(h_{1}+h_{2}\right) e^{-\xi \lambda_{i}}$, with $h_{1}, h_{2} \in \mathbb{R}$, and by reordering the coefficients in the representation (47).

Remark 4 Conditioning: When constructing matrix $M$, the involved inner product produces linear combinations of expressions of the form $1 /\left(\beta_{k}+\beta_{l}\right)$, with $\beta_{k}, \beta_{l}>0$. This implies that the condition number of matrix $M$ rapidly increases with the number of basis terms. In fact, it has a similar behaviour (in terms of the condition number) to the so called Hilbert matrices. In order to avoid numerical instabilities in the method, it is necessary to restrict the total number of basis terms. However, such restriction is not an impediment to obtain accurate approximations, since a relative small number of basis terms is sufficient for such purpose (see numerical results on Section 6.4).

Remark 5 Limitations: A restriction of EIHTM is that it cannot be immediately extended to the AC case. To illustrate this limitation, lets focus on the $i$-th layer, where the spectral solutions is given by a linear combination involving terms of the form [8]:

$\frac{1}{\sqrt{\xi^{2}-k_{i}^{2}}} c_{i j}\left(\xi, z_{\mathrm{Tx}}\right) e^{-\sqrt{\xi^{2}-k_{i}^{2}}(a z+b)}$

where $k_{i}$ denotes a constant wave number that depends upon the considered wave polarization. If the spectral coefficients $c_{i j}\left(\xi, z_{\mathrm{Tx}}\right)$ can be approximated by linear combinations of exponential expressions of the form $e^{-\sqrt{\xi^{2}-k_{i}^{2}} \lambda_{l}}$, for astutely chosen values of $\lambda_{l}$, then the approximated counterpart of (57) will be quadraturefree invertible. However, it will requiere to perform a least-square approximation layer by layer for each position of the tool, giving rise to a very impractical method.

\subsection{Computational Cost Estimates for the EIHTM}

The computational cost in the EIHTM method involves two steps: (1) a pre-process related to the spectral approximation of $\tilde{A}^{-1}(\xi)$ (line 4 in Algorithm 1), and (2) a post-process for the evaluation of the solution (lines 6 and 7 of Algorithm 1).

The computational cost in the pre-process is proportional to the cost of constructing $\tilde{Y}(\xi)$ (see Equation (52)). In fact, once $\tilde{Y}(\xi)$ is already computed, the cost of estimating $C^{-1}$ plus the cost of computing the linear representation for $\tilde{A}^{-T}(\xi)$ given in (47) is relatively small when comparing with the cost of constructing $\tilde{Y}(\xi)$, and thus, it can be neglected.

Construction of $\tilde{Y}(\xi)$ requires solving a Least Squares problem (54), whose cost can be split into three contributions. The first contribution, denoted by $T_{M}$, is related to the cost of constructing matrix $M$. Since $A(\xi)$ is sparse, the number of nonzero entries in each column of $\mathrm{M}$ is bounded by a constant (that does not depend on the number of layers) multiplied by the number of exponential terms $\left(N_{\exp }=m\right)$. Thus, noting that we have $2 \mathrm{~nm}$ basis elements (see (51)), and the number of layers is equal to $N_{\text {layer }}=n+1$, it holds:

$T_{M}=\mathcal{O}\left(N_{\text {layer }} \cdot N_{\exp }^{2}\right)$.

The second contribution, denoted by $T_{\text {rhs }}$, corresponds to the computation of the right-hand side vectors $\mathbf{h}_{i}$, $i=1, \ldots, 2 n$. By the same orthogonality arguments, the cost of computing a single $\mathbf{h}_{i}$ is proportional to $N_{\exp }$, and since it needs to be solved $2 n$ times, we have:

$T_{\text {rhs }}=\mathcal{O}\left(N_{\text {layer }} \cdot N_{\text {exp }}\right)$.

The third cost contribution, denoted by $T_{\text {lsinv }}$, corresponds to the inversion procedure when obtaining the coefficient vectors $\mathbf{s}_{i}, i=1, \ldots, 2 n$. By orthogonality properties, matrix $M$ has a banded structure with bandwidth proportional to $N_{\text {exp }}$. The cost of LU factorization is given by $\mathcal{O}\left(N_{\text {layer }} \cdot N_{\exp }^{3}\right)$, while the cost of a single backward substitution is $\mathcal{O}\left(N_{\text {layer }} \cdot N_{\text {exp }}^{2}\right)$ (cf. [16]). Since the backward substitution procedure needs to be performed $2 n$ times (the LU factorization only needs to be computed once since it can be reutilized), it holds:

$T_{\text {lsinv }}=\mathcal{O}\left(N_{\text {layer }} \cdot N_{\exp }^{3}\right)+\mathcal{O}\left(N_{\text {layer }}^{2} \cdot N_{\exp }^{2}\right)$.

On the other hand, the computational cost for the postprocess, denoted by $T_{\mathrm{ev}}$, corresponds to the analytic evaluation of (18). Since $u_{p}$ is analytic, and the product $\tilde{A}^{-T}(\xi) \mathbf{r}\left(\xi, z_{\mathrm{Tx}}\right)$ does not depend on the number of layers, it can be easily demonstrated that $T_{\mathrm{ev}}$ is given by:

$T_{\text {ev }}=\mathcal{O}\left(N_{\text {exp }} \cdot N_{\text {pos }} \cdot N_{\mathrm{Tx}} \cdot N_{\mathrm{Rx}}\right)$,

where $N_{\text {pos }}$ denotes the number of tool positions, $N_{\text {Tx }}$ the number of transmitters, and $N_{\mathrm{Rx}}$ the number of receivers. 


\section{Computation of Derivatives by using the EIHTM}

Since the derivatives of the solution, in terms of the resistivities, are not depending on the Hankel variable, as an alternative to expression (9), we can also obtain the derivatives as an inverse Hankel transform of derivatives in Hankel space. The advantage of this estimation is that the Hankel inversion is also computed analytically.

For a given resistivity $\rho_{j}$, we need to compute $\partial_{\rho_{j}} u=\partial_{\rho_{j}} u_{p}+\partial_{\rho_{j}} u_{s}=\left(\partial_{\sigma_{j}} u_{p}+\partial_{\sigma_{j}} u_{s}\right) \partial_{\rho_{j}} \sigma_{j}$.

Since $\partial_{\rho_{j}} \sigma_{j}=-\rho_{j}^{-2}$, and $\partial_{\rho_{j}} u_{p}$ can be computed analytically, we just need to estimate

$\partial_{\sigma_{j}} u_{s}=\mathcal{H}^{-1}\left(\partial_{\sigma_{j}} \hat{u}_{s}\right)$,

where $\mathcal{H}^{-1}$ denotes the inverse Hankel transform. From the local representation of $\hat{u}_{s}(14)$, we have

$$
\begin{aligned}
\left.\partial_{\sigma_{j}} \hat{u}_{s}\right|_{\Omega_{i}} & =\frac{1}{4 \pi \xi} \partial_{\sigma_{j}} c_{i 1}\left(\xi, z_{\mathrm{Tx}}\right) e^{-\xi\left(z_{i}-z\right)} \\
& +\frac{1}{4 \pi \xi} \partial_{\sigma_{j}} c_{i 2}\left(\xi, z_{\mathrm{Tx}}\right) e^{-\xi\left(z-z_{i-1}\right)} .
\end{aligned}
$$

In order to compute the derivatives of the spectral functions $c_{i 1}(\xi)$ and $c_{i 2}(\xi)$, we can use the approximation $\mathbf{c}\left(\xi, z_{\mathrm{Tx}}\right) \approx \tilde{A}^{-T}(\xi) \mathbf{r}\left(\xi, z_{\mathrm{Tx}}\right)$, obtaining:

$$
\begin{aligned}
\partial_{\sigma_{j}} \mathbf{c}\left(\xi, z_{\mathrm{Tx}}\right) & \approx\left(\partial_{\sigma_{j}} \tilde{A}^{-T}(\xi)\right) \mathbf{r}\left(\xi, z_{\mathrm{Tx}}\right) \\
& +\tilde{A}^{-T}(\xi)\left(\partial_{\sigma_{j}} \mathbf{r}\left(\xi, z_{\mathrm{Tx}}\right)\right) .
\end{aligned}
$$

Remark 6 The second expression in the right-hand side of equation (65) is different from zero only when $\sigma_{j}=$ $\sigma_{\mathrm{Tx}}$, and in such situation, $\partial_{\sigma_{j}} \mathbf{r}\left(\xi, z_{\mathrm{Tx}}\right)$ contains (in the worst case) just two non-zero terms.

An approximation for $\partial_{\sigma_{j}} \tilde{A}^{-T}(\xi)$ can be recovered by differentiating relation (46), obtaining:

$$
\begin{aligned}
\partial_{\sigma_{j}} \tilde{A}^{-T}(\xi) & =\left(I_{d}-\tilde{Y}(\xi)\right) \partial_{\sigma_{j}} C^{-1} \\
& -\left(\partial_{\sigma_{j}} \tilde{Y}(\xi)\right) C^{-1} .
\end{aligned}
$$

The derivative of $C^{-1}$ is computed analytically, and it is given by:

$\partial_{\sigma_{j}} C^{-1}=-C^{-1}\left(\partial_{\sigma_{j}} C\right) C^{-1}$.

Thus, it only remains to approximate $\partial_{\sigma_{j}} \tilde{Y}(\xi)$. This can be performed in two different ways:

1. $h$-derivative approximation: For a given $h>0$ sufficiently small, we can approximate it as:

$\partial_{\sigma_{j}} \tilde{Y}(\xi) \approx \frac{1}{h}\left(\tilde{Y}_{\sigma_{j}+h}(\xi)-\tilde{Y}(\xi)\right)$,

where $\tilde{Y}_{\sigma_{j}+h}(\xi)$ denotes the exponential approximation obtained from problem (50) after replacing the dependency of $\sigma_{j}$ by $\sigma_{j}+h$.
2. Least Squares approximation: By derivating relation (45), we conclude that:

$$
\begin{aligned}
A^{T}(\xi)\left(\partial_{\sigma_{j}} \tilde{Y}(\xi)\right) & =\partial_{\sigma_{j}} D(\xi) \\
& -\left(\partial_{\sigma_{j}} A^{T}(\xi)\right) \tilde{Y}(\xi)
\end{aligned}
$$

Then, it can be approximated by solving Least Squares problem (50) after modifying the righthand side term.

The $h$-derivative approximation requires to redefine the $M$ matrix for any resistivity (see Equation (54)). However, it only involves a modification of the entries depending on $\sigma_{j}$. This implies that the computational cost is smaller than when using directly Equation (9). The Least Squares approximation requires to redefine the right-hand side term in Equation (54), but it has the advantage that it is $h$-independent. In particular, it is unnecessary to redefine matrix $M$, since the same exponential basis is used for all the resistivities.

Remark 7 Note that an immediate approximation for $\partial_{\sigma_{j}} \tilde{A}^{-T}(\xi)$ can be performed by taking the derivative in the relation $A^{T}(\xi) \tilde{A}^{-T}(\xi) \approx I_{d}$, obtaining:

$\partial_{\sigma_{j}} \tilde{A}^{-T}(\xi) \approx-\tilde{A}^{-T}(\xi)\left(\partial_{\sigma_{j}} A^{T}(\xi)\right) \tilde{A}^{-T}(\xi)$.

Even if $\partial_{\sigma_{j}} A^{T}(\xi)$ is sparse, the main limitation of this approximation is that the number of exponential terms per position of the resulting matrix can increase as $\mathrm{m}^{2}$ (with $m$ being the number of exponential terms). This, in practice, becomes rather expensive.

\section{Numerical examples}

\begin{tabular}{|c|c|c|}
\hline $\begin{array}{c}\text { Logging } \\
\text { Instrument }\end{array}$ & Nr. Tx & Nr. Rx \\
\hline Tool 1 & 1 & 1 \\
\hline Tool 2 & 1 & 2 \\
\hline Tool 3 & 2 & 1 \\
\hline Tool 4 & 2 & 2 \\
\hline
\end{tabular}

Table 1: Logging instrument configurations considered in this paper.

We consider four different tool configurations (operating at zero-frequency), as described in Table 1.

Numerical test were implemented in Matlab R2013a, and executed on a single core of a computer equipped with a 2,9 GHz Intel Core i7 processor with 8 GB RAM. 


\subsection{Traditional Semi-analytical solution}

The reference semi-analytical solution is obtained by using a quadrature rule for the inversion of the Hankel representation of $\hat{u}=\hat{u}_{s}+\hat{u}_{p}$, considering the primary field $\hat{u}_{p}$ as the continuous fundamental Hankel solution (Equation (22)). The function "integral" of Matlab is used for the inverse Hankel transform calculation.

\subsection{Relative Error Definitions}

For a given vector of positions $z_{\text {pos }}$ of size $N_{\text {pos }}$, we denote by $m_{\text {ref }}(u)$ and $m_{\text {eihtm }}(u)$ the vectors of the estimated resistivities obtained by considering the reference semi-analytical solution and EIHTM, respectively. We have evaluated the accuracy of the proposed method by considering the following error estimates (in percent).

The Average Relative Error (ARE):

$\mathrm{ARE}=100 \frac{\left\|m_{\mathrm{ref}}(u)-m_{\mathrm{eihtm}}(u)\right\|}{\left\|m_{\mathrm{ref}}(u)\right\|}$,

where $\|\cdot\|$ denotes the $l^{2}$-norm.

The Pointwise Relative Error (PRE) at the $i$-th position:

$\operatorname{PRE}^{i}=100 \frac{\left|m_{\mathrm{ref}}^{i}(u)-m_{\mathrm{eihtm}}^{i}(u)\right|}{\left|m_{\mathrm{ref}}^{i}(u)\right|}$,

with $m_{\text {ref }}^{i}(u)$ (resp. $\left.m_{\text {eihtm }}^{i}(u)\right)$ denoting the $i$-th coordinate of vector $m_{\text {ref }}(u)$ (resp. $\left.m_{\text {eihtm }}(u)\right)$.

\subsection{Model problems and EIHTM assumptions}

For the numerical examples, we consider the unbounded planar isotropic layered media described by the following vector of vertical positions (in meters):

$\mathbf{z}=\{+\infty, 6,2,1.2,0,-3.8,-4,-5.5,-5.85,-6,-\infty\}$,

with several cases for the values of resistivities (in $\Omega \cdot \mathrm{m}$ ), as illustrated in Table 2 .

In some situations, we consider a different vertical position vector and its formation, which are properly detailed when it corresponds.

In all simulations, we assume that the trajectory is fixed and determined by a given dip angle, and we obtain measurements recorded at equidistant tool positions between $7 \mathrm{~m}$ and $-7 \mathrm{~m}$ (in $z$ ).

To obtain the approximation by using EIHTM, we use an even number of exponential terms $N_{\text {exp }}$. When formation layers exhibit different sizes, we consider
$N_{\min }=N_{\max }=N_{\exp } / 2$ by following the definition given in equation (49). For the particular case when all layers have equal size, the definition must be understood as $N_{\min }=N_{\exp }$ and $N_{\max }=0$.

\subsection{Verification}

As a first example, we compute the apparent resistivity by considering 400 equidistant tool positions. To obtain the approximation of the inverse spectral matrix $\tilde{A}^{-1}(\xi)$ in EIHTM, we consider $N_{\exp }=14$ exponential terms.

Figures 3 and 4 describe the approximations obtained by considering dip angles of $15^{\circ}$ and $75^{\circ}$ for Formation A (Table 2). Figure 5 describes the approximations with dip angle of $15^{\circ}$ and Formation B (Table 2). We display the apparent resistivity in log scale versus the true vertical depth.

In all cases, an accurate approximation in average relative error (ARE) is obtained. Indeed, the worst approximation exhibits an ARE equal to $0.11 \%$ (Figure 3(a)). Moreover, ARE is almost insentisive to the dip angle (compare figures 3 and 4).

The maximum pointwise relative error (PRE) is obtained for the Tool 2 configuration in the Formation $\mathrm{B}$, and it is equal to $33.12 \%$ (Figure $5(\mathrm{~b})$ ). However, a maximum PRE of $33.12 \%$ is indeed a rather accurate approximation estimate as it can be appreciated in Figure 6 , where we compare the apparent resistivity for the Tool 2 configuration and its PRE in log scale.

\subsection{Error analysis}

For a dip angle of $15^{\circ}$ and Formation A, Figure 7 displays the average relative error (ARE) as a function of the number of tool positions. Different lines correspond to different numbers of exponential functions $\left(N_{\exp }\right)$ used for the approximation employed by the EIHTM. The ARE remains below $0.3 \%$ for all tool configurations. For the configurations of Tools 2 and 4 (figures $7(\mathrm{~b})$ and $7(\mathrm{~d}))$, some peaks are observed when the smallest number of exponential terms $\left(N_{\exp }=10\right)$ is considered. These peaks are attenuated by increasing the number of exponential terms, showing also independency in the number of tool positions. The smallest variation is observed in Tool 3 configuration (Figure $7(\mathrm{c}))$. Similar behaviour is obtained in Formation B (Figure 8).

We notice that for engineering purposes, and especially for inversion, the obtained accuracy is sufficient, and the proposed method is robust. 


\begin{tabular}{|c|c|c|c|c|c|c|c|c|c|c|}
\hline $\begin{array}{c}\text { Formation } \\
\text { type }(\Omega \cdot \mathrm{m})\end{array}$ & $\rho_{1}$ & $\rho_{2}$ & $\rho_{3}$ & $\rho_{4}$ & $\rho_{5}$ & $\rho_{6}$ & $\rho_{7}$ & $\rho_{8}$ & $\rho_{9}$ & $\rho_{10}$ \\
\hline $\mathrm{A}$ & 1 & 100 & 1 & 100 & 1 & 100 & 1 & 100 & 1 & 100 \\
\hline $\mathrm{B}$ & 1 & 1000 & 1 & 1000 & 1 & 1000 & 1 & 1000 & 1 & 1000 \\
\hline
\end{tabular}

Table 2: Formation resistivities in $\Omega \cdot \mathrm{m}$ considered for simulations.

\subsection{Cost comparison}

In this section, we compare computational times for the classical semi-analytical method (CSAM) vs. those obtained with the proposed EIHTM. For the CSAM, we select a computational time of a single evaluation equal to $0.001 \mathrm{~s}$, which is 10 times smaller than the computational time referenced for the $\mathrm{AC}$ case in [5] and (in average) 4.8 times smaller than the time required by using the integral function of Matlab, which we know is suboptimal.

For a dip angle of $15^{\circ}$ and Formation A, Figure 9 shows the computational time in seconds required in EIHTM with $N_{\exp }=10$ as a function of the number of tool positions. Similar results with $N_{\exp }=16$ as described in Figure 10. From both figures we conclude that the total simulation time in EIHTM becomes significantly smaller than the reference time in CSAM for all tool configurations. This implies a considerable time reduction when the total number of measurements $\left(N_{\mathrm{pos}} \cdot N_{\mathrm{Tx}} \cdot N_{\mathrm{Rx}}\right)$ is large. For instance, when considering the Tool 4 configuration with $N_{\text {exp }}=16$, the total computational time required by EIHTM in order to simulate 400 tool positions is approximately $0.3 \mathrm{~s}$, which is equivalent to $18.75 \%$ of the reference time spent by traditional semi-analytical methods $(1.6 \mathrm{~s})$. The computational time required in the inversion procedure is independent of the tool configuration, since its dependency is only given by the formation properties and the number of exponential terms (see equations (58), (59) and (60)). Even if the computational time of the pre-process grows as a quadratic function of $N_{\exp }$, a relative small difference is obtained when comparing with the computational time required with $N_{\exp }=10$ and $N_{\text {exp }}=16$. Thus, EITHM delivers accurate results, while maintaining the fast resolution property.

Figure 11 shows the computational time required by EIHTM with $N_{\exp }=12$ as a function of $N_{\text {layer }}$, preserving the end layer length values given by the vector of vertical positions (73), and considering the remaining layers lengths with equal size. For the resistitivities, a formation following the structure of Formation A (Table 2) is assumed, i.e. alternations of the values $1 \Omega \cdot \mathrm{m}$ and $100 \Omega \cdot \mathrm{m}$. The computational cost independency of the post-process, with respect to $N_{\text {layer }}$ is clearly evidenced. Indeed, when considering a par- ticular tool configuration, the same slope is obtained for all $N_{\text {layer, }}$ validating the post-process time estimation given in equation (61). The dependency in $N_{\text {layer }}$ is given in the pre-process computation, which grows quadratically in terms of $N_{\text {layer }}$. However, this implies only a small variation in the total time when increasing the number of layers, making possible the simulation of a large number of tool positions in a very fast way. For instance, with $N_{\text {layer }}=30$ and 2500 positions, for the tool 4 configuration, the total time required for EIHTM is less than $1.5 \mathrm{~s}$ vs $30 \mathrm{~s}$ required by CSAM (a reduction by a factor of 20 ).

In the next examples, we describe the computational time required in the pre-process of EIHTM (see equations (58), (59) and (60)). Since the computational time is independent of the layer lengths, we consider (for simplicity) that the media is composed of layers with the same length, and resistivities formations following the structure of Formation A.

Figure 12 shows the computational time required by EIHTM as a function of $N_{\text {exp }}$ for a model composed of 12 equidistant layers. Figure 13 shows the computational time required by EIHTM with $N_{\exp }=10$ as a function of $N_{\text {layer. }}$ In both cases, we observe that the best fitting curves reproduce the behaviour estimated in section 4.5 , validating the cost estimates.

\section{Conclusions}

We propose a new semi-analytical method (EIHTM) for the fast simulation and inversion of $1.5 \mathrm{D}$ direct current borehole measurements. The EIHTM requires a pre-process step that is expensive when compared with a single evaluation obtained by using a classical semianalytical method. However, when considering a large number of logging positions, the total time of the EIHTM is considerably smaller than that of classical semianalytical methods. When solving the inverse problem, the EIHTM also allows to use alternatives to the classical discrete definition of the derivative for the Jacobian estimation and, in particular, we provide two explicit representations.

For the sake of simplicity, the method was introduced only for isotropic media. However, it can be easily extended to the TI case by incorporating an adequate change of variables in the Hankel representation of the 
solution.

Several numerical tests for the forward problem were performed to validate the robustness, accuracy, and efficiency of the method. We considered different tool configurations and layer lengths with high resistivity contrasts. For all considered tool configurations, accurate solutions were obtained for relatively small numbers of exponential terms. Numerical results also showed that the efficiency of the method is independent of the dip angle. For a sufficiently large number of exponential terms, the approximation error becomes independent of the tool position, making it a robust approximation method.

We derived explicit estimates for the computational cost of EIHTM, which we further validated via numerical experimentation. We conclude that, for a large number of measurements, the EITHM method is faster than any existing semi-analytical method, and its computational time divided by that spent by classical semianalytical methods is maximized as we increase the number of simulated measurements.

The principal limitation of EITHM is that the extension to the AC case seems challenging, since in such situation, the exponential analytic solution of the ODE Hankel representation depends nonlinearly upon the frequency in the exponential representations. As a future work, we shall investigate more in detail the feseability of a practical method for 1.5D AC simulations considering quadrature-free expansions defined over each layer (see Remark 5).

Acknowledgements Sergio Rojas acknowledge support from CONICYT-Chile through $\mathrm{PhD}$ fellowship program and project Anillo ACT1112 "Stochastic Analysis Research Network". Ignacio Muga was partially funded by the Ibero-American Project CYTED 2011 (P711RT0278) and CONICYT-Chile through project Anillo ACT1118 (ANANUM). David Pardo has received funding from the European Union's Horizon 2020 research and innovation programme under the Marie Sklodowska-Curie grant agreement No 644602, the Project of the Spanish Ministry of Economy and Competitiveness with reference MTM2013-40824-P, the BCAM "Severo Ochoa" accreditation of excellence SEV-20130323, and the Basque Government through the BERC 20142017 program and the Consolidated Research Group Grant IT649-13 on "Mathematical Modeling, Simulation, and Industrial Applications (M2SI)".

\section{References}

1. Avdeev, D.B., Kuvshinov, A.V., Pankratov, O.V., Newman, G.A.: Three-dimensional induction logging problems, Part I: An integral equation solution and model comparisons. Geophysics 67(2), 413-426 (2002)

2. Chew, W.C., Chen, S.Y.: Response of a point source embedded in a layered medium. Antennas and Wireless Propagation Letters, IEEE 2(1), 254-258 (2003)
3. Davydycheva, S., Druskin, V., Habashy, T., et al.: Finitedifference scheme for electromagnetic logging in 3D anisotropic media. In: 2004 SEG Annual Meeting. Society of Exploration Geophysicists (2004)

4. Druskin, V.L., Knizhnerman, L., Lee, P.: New spectral Lanczos decomposition method for induction modeling in arbitrary 3-D geometry. Geophysics 64(3), 701-706 (1999)

5. Dyatlov, G., Onegova, E., Dashevsky, Y.: Efficient 2.5 D electromagnetic modeling using boundary integral equations. Geophysics 80(3), E163-E173 (2015)

6. Gajda-Zagórska, E., Schaefer, R., Smołka, M., Paszyński, M., Pardo, D.: A hybrid method for inversion of 3D DC resistivity logging measurements. Natural Computing pp. 1-20 (2014)

7. Ijasan, O., Torres-Verdín, C., Preeg, W.E.: Inversionbased petrophysical interpretation of logging-whiledrilling nuclear and resistivity measurements. Geophysics 78(6), D473-D489 (2013)

8. Kong, J.: Electromagnetic fields due to dipole antennas over stratified anisotropic media. Geophysics 37(6), 985$996(1972)$

9. Løseth, L., Ursin, B.: Electromagnetic fields in planarly layered anisotropic media. Geophysical Journal International 170(1), 44-80 (2007)

10. Merchant, G., Strickland, R.W., Jackson, C., et al.: Enhanced resolution LWD resistivity logs using a new inversion technique. In: SPWLA 37th Annual Logging Symposium. Society of Petrophysicists and Well-Log Analysts (1996)

11. Meyer, W.H., et al.: Inversion of $2 \mathrm{MHz}$ Propagation Resistivity Logs in Dipping Thin Beds. In: SPWLA 34th Annual Logging Symposium. Society of Petrophysicists and Well-Log Analysts (1993)

12. Nam, M.J., Pardo, D., Torres-Verdín, C.: Simulation of dual-laterolog measurements in dipping, invaded and anisotropic formations using a Fourier series expansion in a non-orthogonal system of coordinates and a selfadaptive hp-finite element method. Geophysics $\mathbf{7 4}(1)$ (2008)

13. Nam, M.J., Pardo, D., Torres-Verdín, C.: Assessment of Delaware and Groningen effects on dual-laterolog measurements with a self-adaptive hp finite-element method. Geophysics 75(6), F143-F149 (2010)

14. Newman, G.A., Alumbaugh, D.L.: Three-dimensional induction logging problems, Part 2: A finite-difference solution. Geophysics 67(2), 484-491 (2002)

15. Papoulis, A.: Systems and transforms with applications in optics. McGraw-Hill Series in System Science, Malabar: Krieger, 19681 (1968)

16. Pardo, D., Paszynski, M., Collier, N., Alvarez, J., Dalcin, L., Calo, V.M.: A survey on direct solvers for galerkin methods. SeMA Journal 57(1), 107-134 (2012)

17. Pardo, D., Paszynski, M., Torres-Verdín, C., Demkowicz, L.: Simulations of 3D DC borehole resistivity measurements with a goal-oriented hp finite-element method. Part I: laterolog and LWD. Journal of the Serbian Society for Computational Mechanics 1, 62-73 (2007)

18. Pardo, D., Torres-Verdín, C.: Fast 1D inversion of logging-while-drilling resistivity measurements for improved estimation of formation resistivity in high-angle and horizontal wells. Geophysics 80(2), E111-E124 (2015)

19. Pardo, D., Torres-Verdín, C., Nam, M., Paszynski, M., Calo, V.: Fourier series expansion in a non-orthogonal system of coordinates for the simulation of 3D alternating current borehole resistivity measurements. Computer 
Methods in Applied Mechanics and Engineering 197(45), 3836-3849 (2008)

20. Pardo, D., Torres-Verdín, C., Paszynski, M.: Simulations of 3D DC borehole resistivity measurements with a goal-oriented hp finite-element method. Part II: throughcasing resistivity instruments. Computational Geosciences 12(1), 83-89 (2008)

21. Sjödahl, P., Dahlin, T., Zhou, B.: 2.5 D resistivity modeling of embankment dams to assess influence from geometry and material properties. Geophysics 71(3), G107G114 (2006)

22. Streich, R., Becken, M.: Sensitivity of controlled-source electromagnetic fields in planarly layered media. Geophysical Journal International 187(2), 705-728 (2011)

23. Tai, C.T.: Dyadic Green functions in electromagnetic theory. Institute of Electrical \& Electronics Engineers (IEEE) (1994)

24. Wait, J.R.: The magnetic dipole over the horizontally stratified Earth. Canadian Journal of Physics 29(6), 577$592(1951)$

25. Wang, T., Fang, S.: 3-D electromagnetic anisotropy modeling using finite differences. Geophysics 66(5), 13861398 (2001)

26. Wang, T., Signorelli, J.: Finite-difference modeling of electromagnetic tool response for logging while drilling. Geophysics 69(1), 152-160 (2004)

27. Zhang, J., Mackie, R.L., Madden, T.R.: 3-D resistivity forward modeling and inversion using conjugate gradients. Geophysics 60(5), 1313-1325 (1995)

28. Zhong, L., Li, J., Bhardwaj, A., Shen, L.C., Liu, R.C.: Computation of triaxial induction logging tools in layered anisotropic dipping formations. Geoscience and Remote Sensing, IEEE Transactions on 46(4), 1148-1163 (2008) 


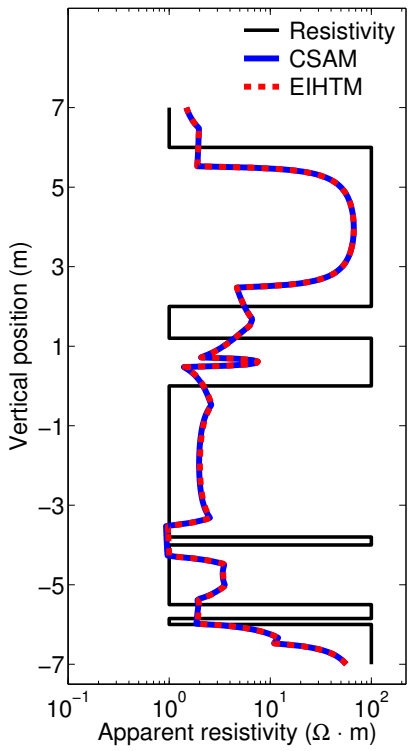

(a) Tool 1 Configuration. ARE $=0.108 \%$. Max PRE $=4.361 \%$.

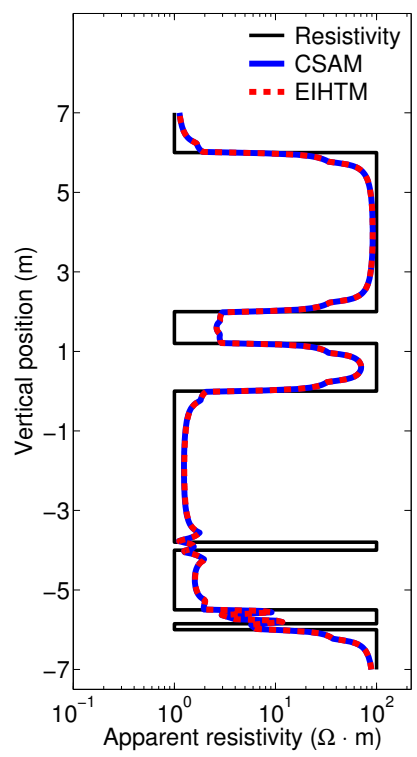

(c) Tool 3 Configuration. $\mathrm{ARE}=0.015 \%$. Max PRE $=$ $0.875 \%$.

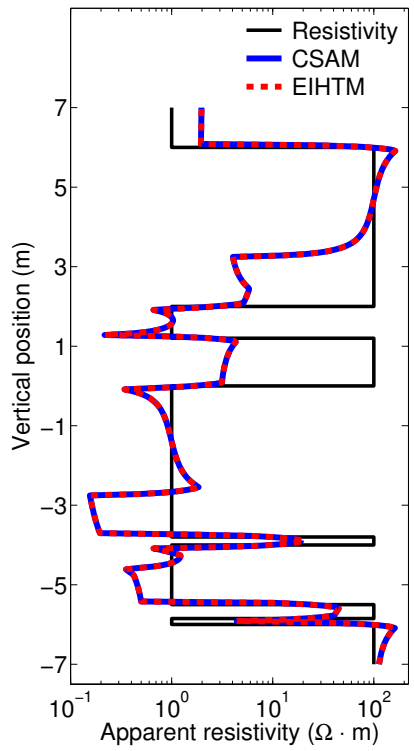

(b) Tool 2 Configuration. $\mathrm{ARE}=0.035 \%$. Max PRE $=$ $15.610 \%$.

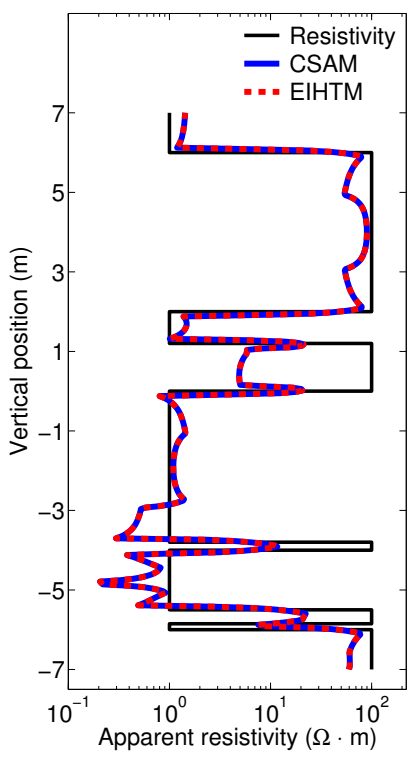

(d) Tool 4 Configuration. $\mathrm{ARE}=0.006 \%$. Max PRE $=$ $0.217 \%$.

Fig. 3: Apparent $(\log )$ resisitivities example. Dip angle $=15^{\circ}$. Formation A. EIHTM with $N_{\exp }=14$. 


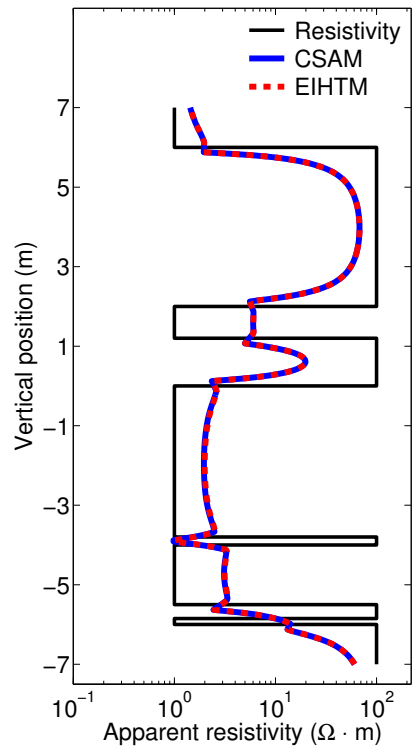

(a) Tool 1 Configuration. $\mathrm{ARE}=0.097 \%$. Max PRE $=$ $4.146 \%$.

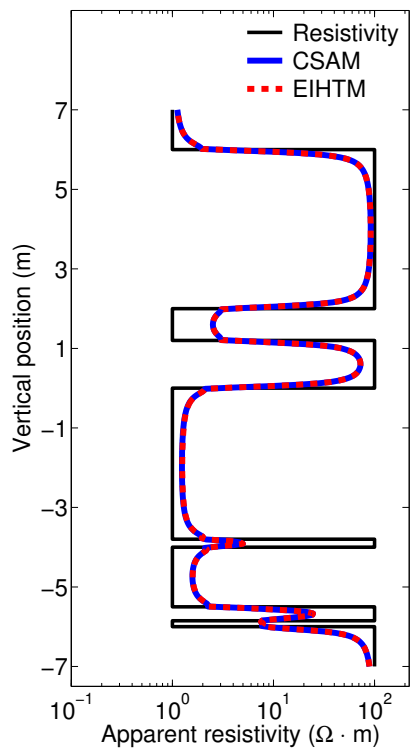

(c) Tool 3 Configuration. $\mathrm{ARE}=0.014 \%$. Max PRE $=$ $0.739 \%$.

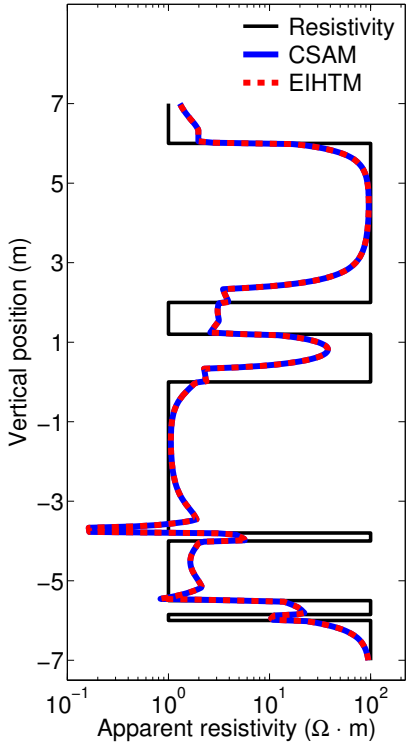

(b) Tool 2 Configuration. $\mathrm{ARE}=0.034 \%$. Max PRE $=$ $4.869 \%$.

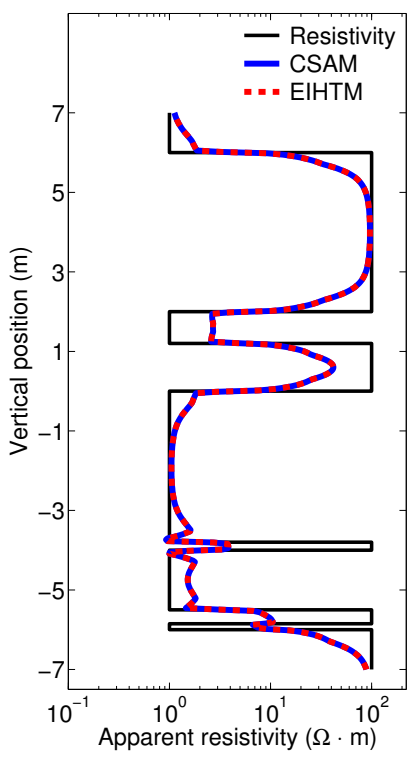

(d) Tool 4 Configuration. $\mathrm{ARE}=0.008 \%$. Max PRE $=$ $0.814 \%$.

Fig. 4: Apparent $(\log )$ resisitivities example. Dip angle $=75^{\circ}$. Formation A. EIHTM with $N_{\exp }=14$. 


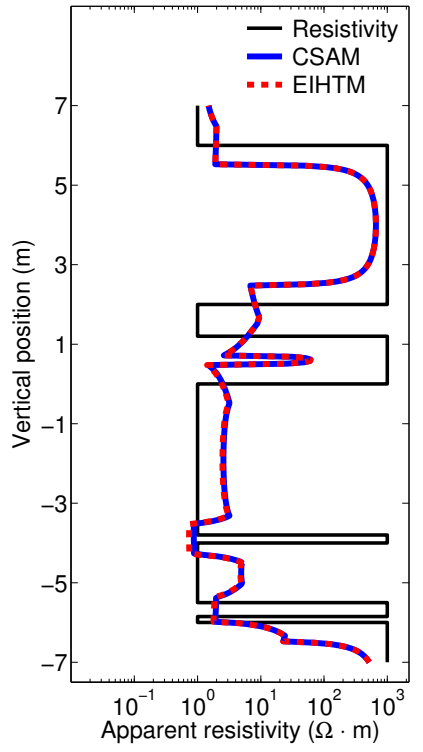

(a) Tool 1 Configuration. $\mathrm{ARE}=0.033 \%$. Max PRE $=$ $15.794 \%$.

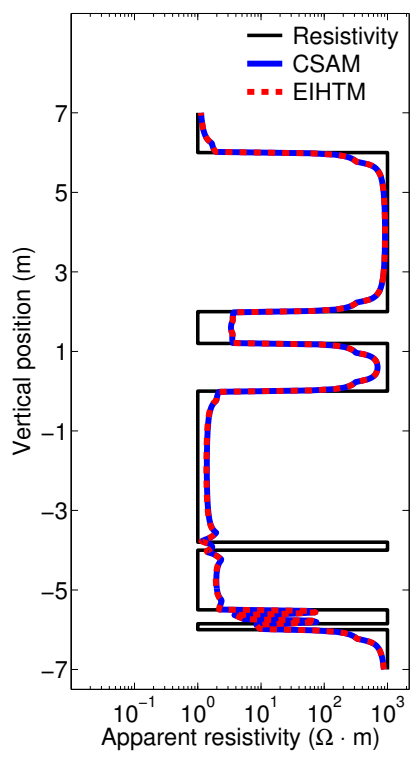

(c) Tool 3 Configuration $\mathrm{ARE}=0.004 \%$. Max PRE $=$ $2.439 \%$.

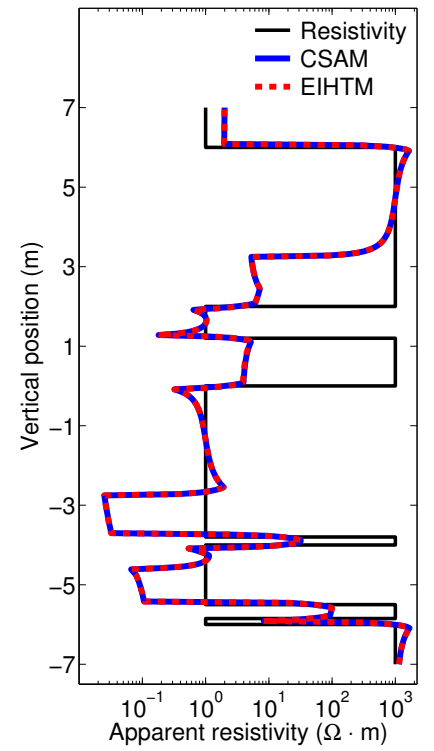

(b) Tool 2 Configuration. $\mathrm{ARE}=0.009 \%$. Max PRE $=$ $33.115 \%$.

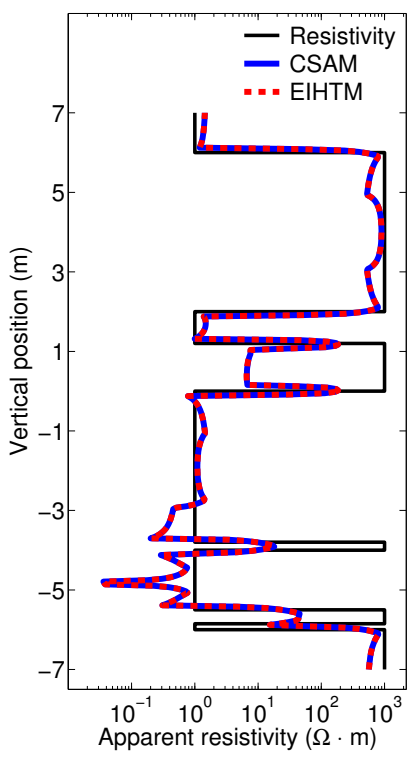

(d) Tool 4 Configuration. $\mathrm{ARE}=0.006 \%$. Max PRE $=$ $1.442 \%$.

Fig. 5: Apparent $(\log )$ resisitivities example. Dip angle $=15^{\circ}$. Formation B. EIHTM with $N_{\exp }=14$. 


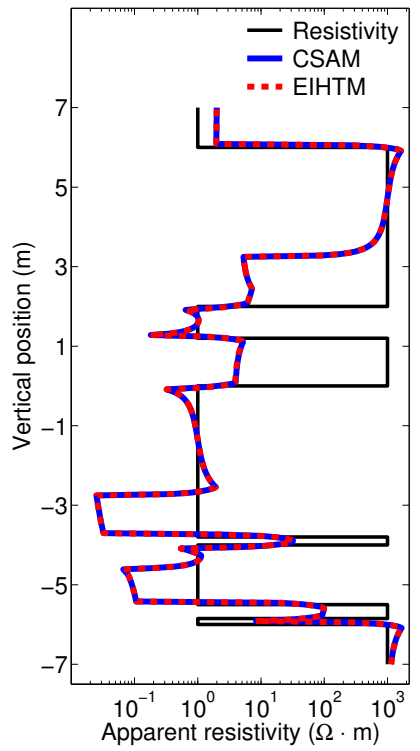

(a) EIHTM with $N_{\exp }=14$. $\mathrm{ARE}=0.009 \%$. Max PRE $=$ $33.115 \%$.

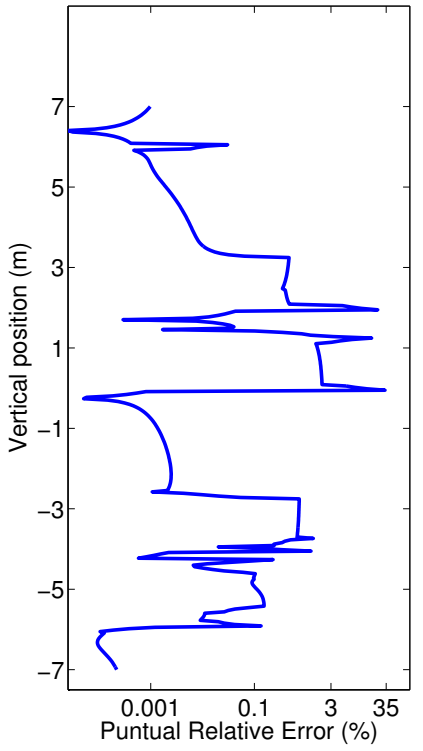

(b) EIHTM PRE.

Fig. 6: Pointwise Relative Error example. Tool 2 configuration. Dip angle $=15^{\circ}$. Formation B.

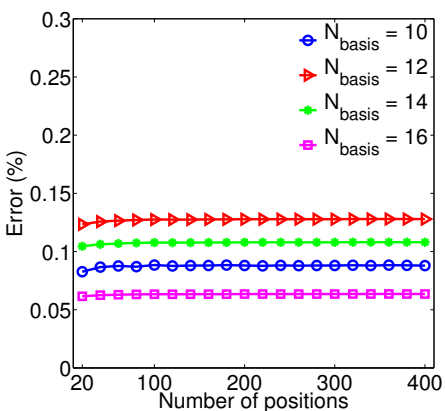

(a) Tool 1 .

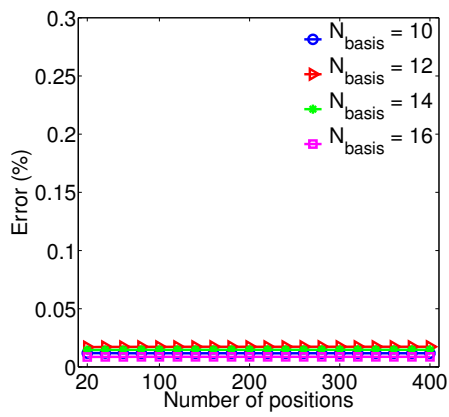

(c) Tool 3.

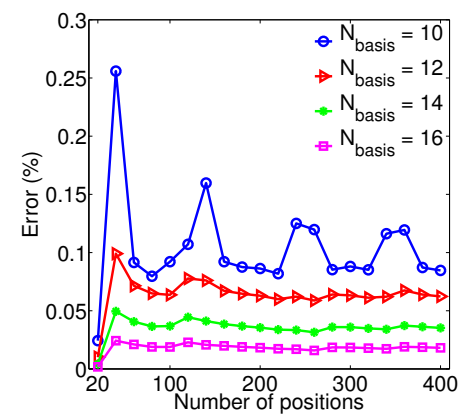

(b) Tool 2 .

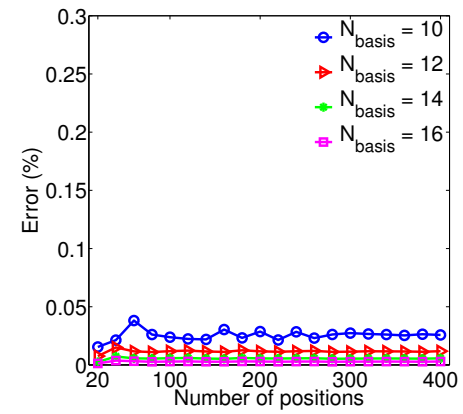

(d) Tool 4.

Fig. 7: Average Relative Error. Dip angle of $15^{\circ}$. Formation A. 


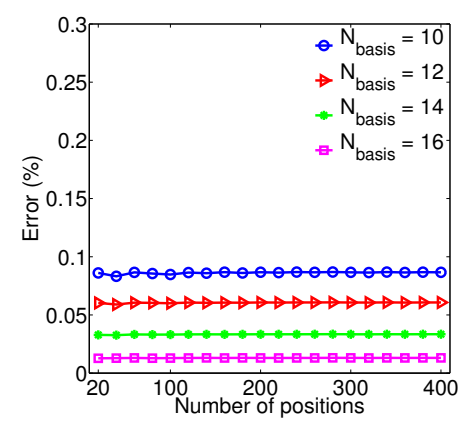

(a) Tool 1.

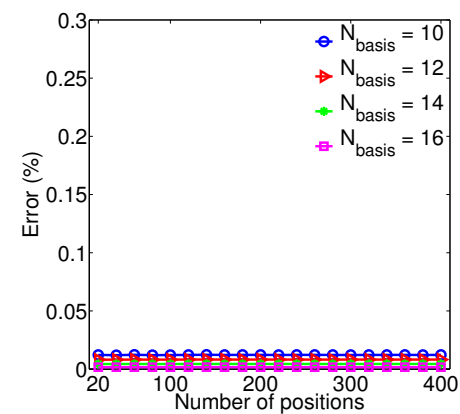

(c) Tool 3.

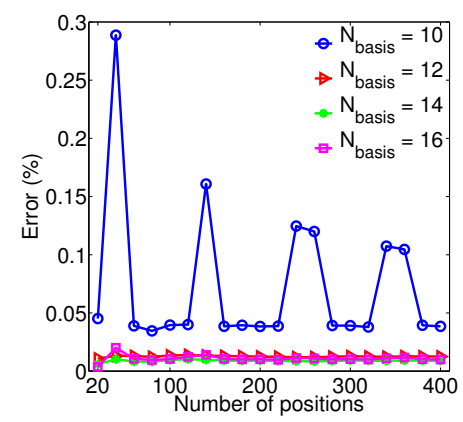

(b) Tool 2 .

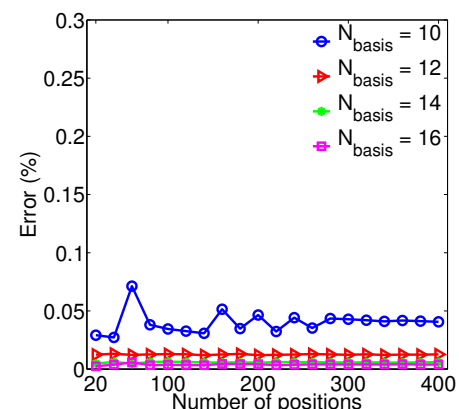

(d) Tool 4 .

Fig. 8: Average Relative Error. Dip angle of $15^{\circ}$. Formation B.

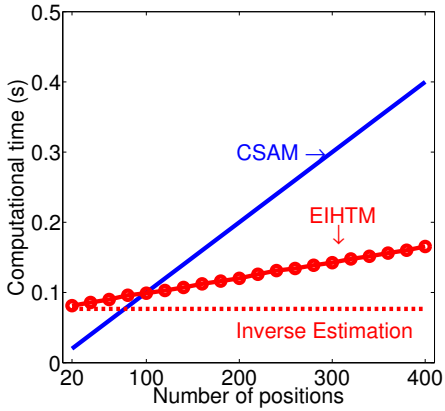

(a) Tool 1.

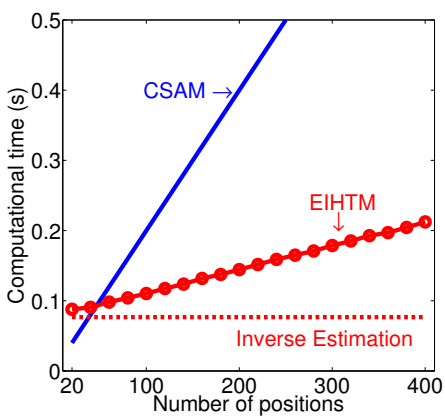

(c) Tool 3.

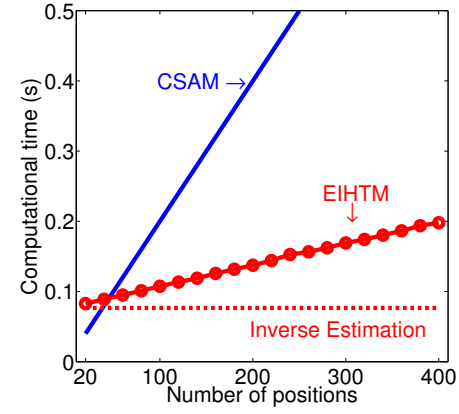

(b) Tool 2.

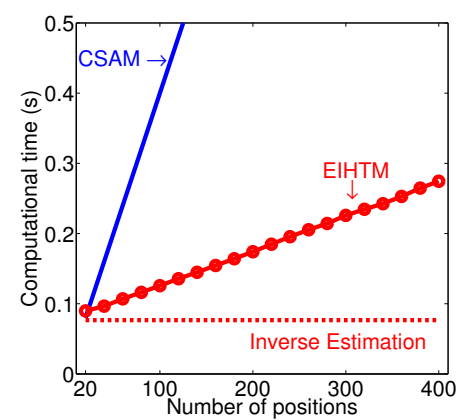

(d) Tool 4 .

Fig. 9: Time (s) comparison between EIHTM (with $N_{\exp }=10$ ), and classical semi-analytical methods (CSAM). Dashed (red) line represents the computational time required by EIHTM in the pre-process, which consists of estimating $A^{-1}(\xi)$. 


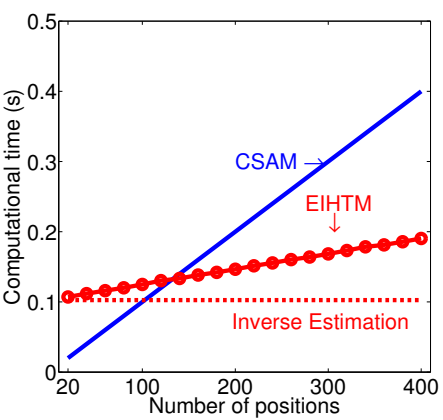

(a) Tool 1.

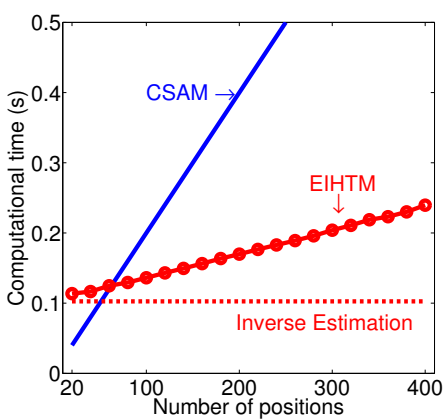

(c) Tool 3.

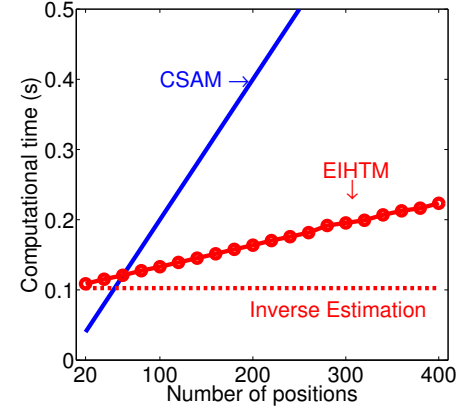

(b) Tool 2.

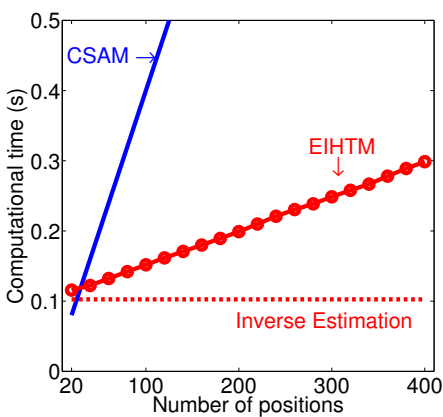

(d) Tool 4.

Fig. 10: Time (s) comparison between EIHTM (with $N_{\exp }=16$ ), and classical semi-analytical methods (CSAM) for different tool configurations. Dashed (red) line represents the computational time required by EIHTM in the which consists of estimating $A^{-1}(\xi)$.

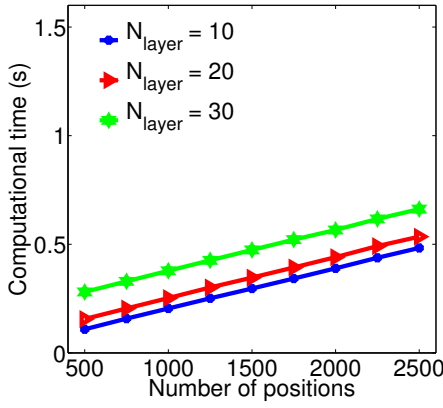

(a) Tool 1.

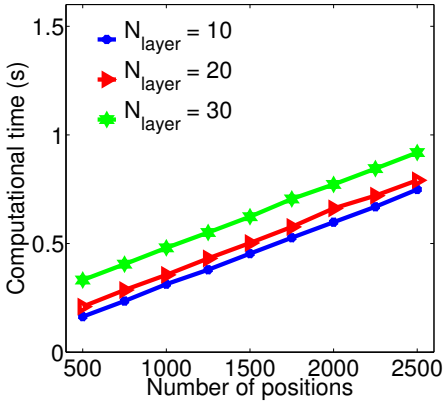

(c) Tool 3.

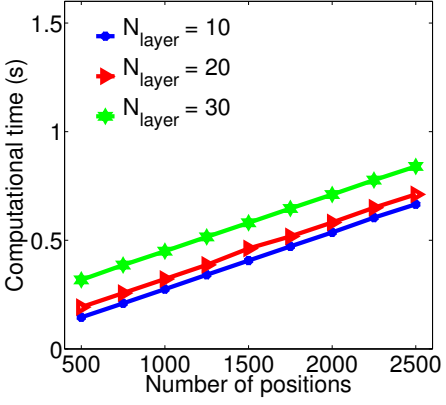

(b) Tool 2.

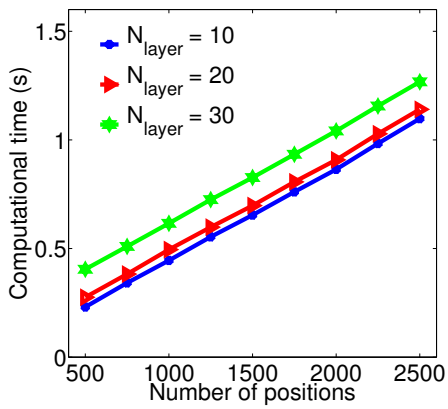

(d) Tool 4

Fig. 11: Time (s) in EIHTM with respect to the number of layers for different tool configurations and number of positions. 


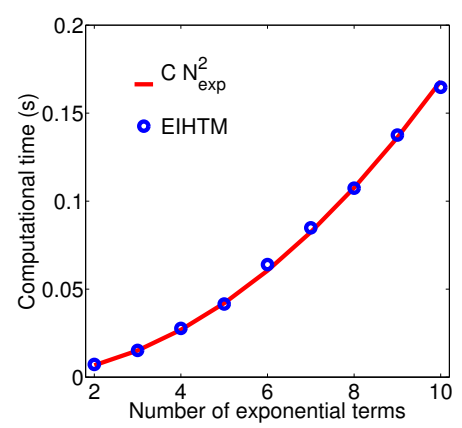

(a) Matrix $M$ construction.

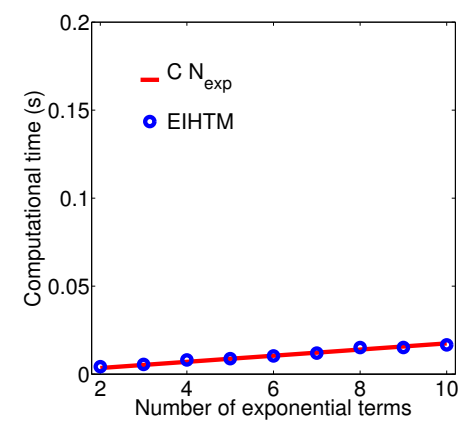

(b) Right-hand side constructions.

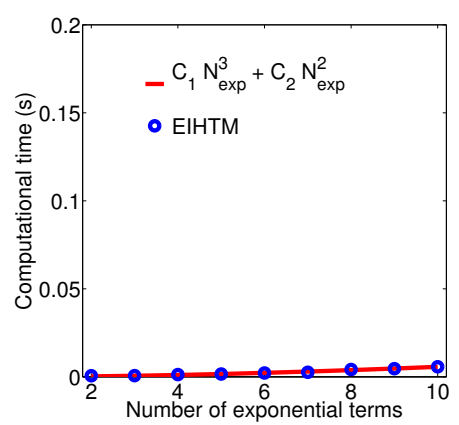

(c) Least squares inversion.

Fig. 12: EIHTM pre-process computational cost example with $N_{\text {layer }}=12$, as a function of variable $N_{\text {exp }}$.

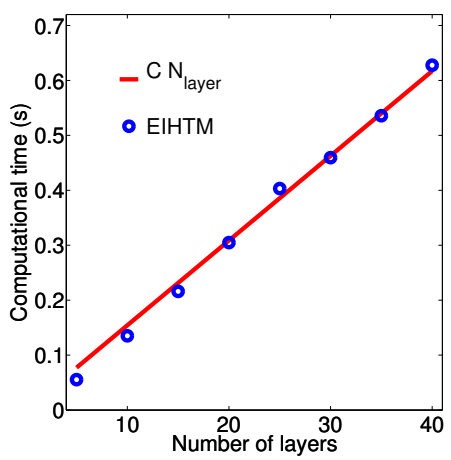

(a) Matrix $M$ construction.

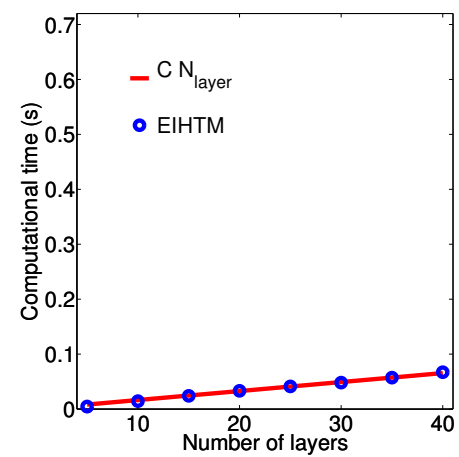

(b) Right-hand side constructions.

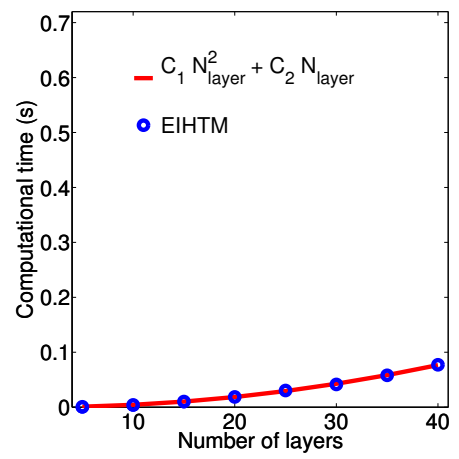

(c) Least squares inversion.

Fig. 13: EIHTM pre-process computational cost example with $N_{\exp }=10$, as a function of variable $N_{\text {layer }}$. 